\title{
Local exact controllability to the trajectories of the Boussinesq system
}

\section{Contrôlabilité exacte locale aux trajectoires du système de Boussinesq}

\author{
S. Guerrero ${ }^{1}$ \\ Dpto. E.D.A.N., University of Sevilla, Aptdo. 1160, 41080 Sevilla, Spain
}

Received 28 July 2004; received in revised form 12 October 2004; accepted 13 January 2005

Available online 20 April 2005

\begin{abstract}
In this paper we deal with the local exact controllability to the trajectories of the Boussinesq system with $N+1$ distributed scalar controls supported in small sets. In a first step, we present a new Carleman inequality for a linearized version of the Boussinesq system, which leads to its null controllability at any time $T>0$. Then, we deduce the desired result concerning the local exact controllability of the (nonlinear) Boussinesq system.

(C) 2006 L'Association Publications de l'Institut Henri Poincaré. Published by Elsevier B.V. All rights reserved
\end{abstract}

\section{Résumé}

Dans ce papier on étudie la contrôlabilité exacte locale aux trajectoires du système de Boussinesq avec $N+1$ contrôles scalaires localisés dans des petits ouverts. Dans un premier temps, on présente une nouvelle inégalité de Carleman pour un système de Boussinesq linéarisé, ce qui nous permet d'établir la contrôlabilité à zéro de ce système pour tout temps $T>0$. On en déduit ensuite la contrôlabilité exacte locale du système (non linéaire) de Boussinesq.

(C) 2006 L'Association Publications de l'Institut Henri Poincaré. Published by Elsevier B.V. All rights reserved

MSC: 34B15; 93B05; 93C10

Keywords: Global Carleman inequalities; Exact controllability; Nonlinear systems

E-mail address: sguerrero@us.es (S. Guerrero).

1 This work has been partially supported by D.G.E.S. (Spain), Grant BFM2000-1317.

0294-1449/\$ - see front matter (C) 2006 L'Association Publications de l'Institut Henri Poincaré. Published by Elsevier B.V. All rights reserved doi:10.1016/j.anihpc.2005.01.002 


\section{Introduction}

Let $\Omega \subset \mathbb{R}^{N}$ ( $N=2$ or 3 ) be a bounded connected open set whose boundary $\partial \Omega$ is regular enough. Let $\omega \subset \Omega$ be a (small) nonempty open subset and let $T>0$. We will use the notation $Q=\Omega \times(0, T)$ and $\Sigma=\partial \Omega \times(0, T)$ and we will denote by $n(x)$ the outward unit normal to $\Omega$ at the point $x \in \partial \Omega$.

We will be concerned with the controlled Boussinesq system

$$
\begin{cases}y_{t}-\Delta y+(y \cdot \nabla) y+\nabla p=\theta e_{N}+v_{1} 1_{\omega} & \text { in } Q, \\ \theta_{t}-\Delta \theta+y \cdot \nabla \theta=v_{2} 1_{\omega} & \text { in } Q, \\ \nabla \cdot y=0 & \text { in } Q, \\ y=0, \quad \theta=0 & \text { on } \Sigma, \\ y(0)=y^{0}, \quad \theta(0)=\theta^{0} & \text { in } \Omega,\end{cases}
$$

where

$$
e_{N}= \begin{cases}(0,1) & \text { if } N=2, \\ (0,0,1) & \text { if } N=3\end{cases}
$$

stands for the gravity vector field. Here, $y=y(x, t)$ represents the velocity of the particles of the fluid, $\theta=\theta(x, t)$ their temperature and $\left(y^{0}, \theta^{0}\right)$ are the initial states, that is to say, the states at time $t=0$. On the other hand, $v_{1}$ and $v_{2}$ stand for control functions which act over the system just through the control domain $\omega$.

The following vector field sets, usual in the context of fluids, will be used along the paper:

$$
H=\left\{w \in L^{2}(\Omega)^{N}: \nabla \cdot w=0 \text { in } \Omega \text { and } w \cdot n=0 \text { on } \partial \Omega\right\}
$$

and

$$
V=\left\{w \in H_{0}^{1}(\Omega)^{N}: \nabla \cdot w=0 \text { in } \Omega\right\} .
$$

In order to introduce the problem we want to solve, we fix a trajectory $(\bar{y}, \bar{\theta})$ (together with certain pressure $\bar{p}$ ), which will be a regular enough solution of a noncontrolled Boussinesq system similar to (1). More precisely, they will be assumed to satisfy

$$
\begin{cases}\bar{y}_{t}-\Delta \bar{y}+(\bar{y} \cdot \nabla) \bar{y}+\nabla \bar{p}=\bar{\theta} e_{N} & \text { in } Q, \\ \bar{\theta}_{t}-\Delta \bar{\theta}+\bar{y} \cdot \nabla \bar{\theta}=0 & \text { in } Q, \\ \nabla \cdot \bar{y}=0 & \text { in } Q, \\ \bar{y}=0, \quad \bar{\theta}=0 & \text { on } \Sigma, \\ \bar{y}(0)=\bar{y}^{0}, \quad \bar{\theta}(0)=\bar{\theta}^{0} & \text { in } \Omega .\end{cases}
$$

We will additionally suppose that they verify the following regularity properties:

$$
\bar{y}_{i}, \bar{\theta} \in L^{\infty}(Q), \quad\left(\bar{y}_{t}\right)_{i}, \bar{\theta}_{t} \in L^{2}\left(0, T ; L^{r}(\Omega)\right), \quad 1 \leqslant i \leqslant N,
$$

with

$$
r> \begin{cases}1 & \text { if } N=2, \\ 6 / 5 & \text { if } N=3 .\end{cases}
$$

As long as the initial conditions are concerned, let us introduce the space

$$
E_{0}= \begin{cases}L^{2}(\Omega)^{2} & \text { if } N=2, \\ L^{4}(\Omega)^{3} \cap H & \text { if } N=3 .\end{cases}
$$

With this notation, we will also suppose that

$$
\left(\bar{y}^{0}, \bar{\theta}^{0}\right) \in E_{0} \times L^{2}(\Omega) .
$$

For system (1), we introduce the concept of local exact controllability to the trajectories at time $t=T$. It consists of finding suitable controls $\left(v_{1}, v_{2}\right)$ such that the corresponding solution of (1) coincides with that of (4) at time $T$. 
More precisely, we will say that the local exact controllability to the trajectories $(\bar{y}, \bar{\theta})$ at time $T$ holds if there exists $\delta>0$ such that, for each initial condition $\left(y^{0}, \theta^{0}\right) \in E_{0} \in L^{2}(\Omega)$ verifying $\left\|\left(y^{0}, \theta^{0}\right)-\left(\bar{y}^{0}, \bar{\theta}^{0}\right)\right\|_{E_{0} \times L^{2}(\Omega)} \leqslant \delta$, there exist two controls $\left(v_{1}, v_{2}\right) \in L^{2}(\omega \times(0, T))^{N+1}$ such that

$$
y(T)=\bar{y}(T) \text { and } \quad \theta(T)=\bar{\theta}(T) \text { in } \Omega .
$$

For the moment, the unique results concerning the controllability of Boussinesq systems have been proved by A.V. Fursikov and O.Yu. Imanuvilov (see [5] and [6]). In these works, they establish the local exact boundary controllability to the trajectories of the Boussinesq system with $N+1$ scalar controls acting over the whole boundary $\partial \Omega$ and the local exact controllability to the same trajectories with $N+1$ scalar distributed controls when $\Omega$ is a torus. More precisely, if we fix a regular trajectory $(\bar{y}, \bar{p}, \bar{\theta})$ and we take $\left(y^{0}, \theta^{0}\right)$ to be close enough (in $V \times H_{0}^{1}(\Omega)$ norm) to $\left(\bar{y}^{0}, \bar{\theta}^{0}\right)$, one can find controls $\left(v_{1}, v_{2}\right) \in L^{2}(\Sigma)^{N+1}$ such that the solution of

$$
\begin{cases}y_{t}-\Delta y+(y \cdot \nabla) y+\nabla p=\theta e_{N} & \text { in } Q, \\ \theta_{t}-\Delta \theta+y \cdot \nabla \theta=0 & \text { in } Q, \\ \nabla \cdot y=0 & \text { in } Q \\ y=v_{1}, \quad \theta=v_{2} & \text { on } \Sigma \\ y(0)=y^{0}, \quad \theta(0)=\theta^{0} & \text { in } \Omega\end{cases}
$$

satisfies (9). By a regular trajectory we mean, for instance (see Remark 1.1 in [5])

$$
(\bar{y}, \bar{\theta}) \in L^{2}\left(0, T ; H^{5 / 2}(\Omega)^{N+1}\right) \cap H^{1}\left(0, T ; H^{1 / 2}(\Omega)^{N+1}\right) .
$$

Analogously, the same result holds when acting with $N$ scalar controls in the movement equation and another one in the heat equation over $\omega$, as long as $\Omega$ is a torus.

The main result of this paper concerns the local exact controllability to the trajectories of system (1) and is presented in the following theorem:

Theorem 1. Let $\left(y^{0}, \theta^{0}\right) \in E_{0} \times L^{2}(\Omega)$ and $T>0$. Then, we have the local exact controllability at time $T$ to the solutions $(\bar{y}, \bar{p}, \bar{\theta})$ of system (4) satisfying (5), i.e., there exists $\delta>0$ such that if $\left\|\left(y^{0}-\bar{y}^{0}, \theta^{0}-\bar{\theta}^{0}\right)\right\|_{E_{0} \times L^{2}(\Omega)} \leqslant \delta$, we can find controls $\left(v_{1}, v_{2}\right) \in L^{2}(\omega \times(0, T))^{N+1}$ such that (9) holds.

Remark 1. In [3], the authors proved the local exact controllability of the Navier-Stokes system

$$
\begin{cases}y_{t}-\Delta y+\nabla \cdot(y \otimes y)+\nabla p=v 1_{\omega} & \text { in } Q, \\ \nabla \cdot y=0 & \text { in } Q, \\ y=0 & \text { on } \Sigma, \\ y(0)=y^{0} & \text { in } \Omega\end{cases}
$$

to the trajectories of the same system which verifies the same regularity hypotheses stated in (5). Therefore, it will be seen in this paper that the presence of a (coupling) heat equation does not impose any further regularity on the trajectories than the ones stated in [3].

Let us now give a sketch of the strategy we will follow to attack our problem:

- We consider the following nonlinear system:

$$
\begin{cases}z_{t}-\Delta z+(z \cdot \nabla) z+(\bar{y} \cdot \nabla) z+(z \cdot \nabla) \bar{y}+\nabla q=\rho e_{N}+v_{1} 1_{\omega} & \text { in } Q, \\ \rho_{t}-\Delta \rho+z \cdot \nabla \rho+\bar{y} \cdot \nabla \rho+\nabla \cdot(\bar{\theta} z)=v_{2} 1_{\omega} & \text { in } Q, \\ \nabla \cdot z=0 & \text { in } Q, \\ z=0, \quad \rho=0 & \text { on } \Sigma, \\ z(0)=z^{0}, \quad \rho(0)=\rho^{0} & \text { in } \Omega .\end{cases}
$$


Then, if there exists $\delta>0$ such that under condition $\left\|\left(z^{0}, \rho^{0}\right)\right\|_{E_{0} \times L^{2}(\Omega)} \leqslant \delta$ we can find two controls $v_{1}$ and $v_{2}$ such that

$$
z_{i}(T)=\rho(T)=0 \quad \text { in } \Omega \quad(1 \leqslant i \leqslant N),
$$

setting $(y, p, \theta)=(z+\bar{y}, q+\bar{p}, \rho+\bar{\theta})$ the local exact controllability to the trajectory $(\bar{y}, \bar{\theta})$ would be established.

Consequently, the local exact controllability result to the trajectory $(\bar{y}, \bar{\theta}$ ) (Theorem 1 ) has been reduced to the local null controllability for the solution $(z, \rho)$ of system (11). The proof of this result will be the goal of Section 4 .

- In order to prove this local result, we will employ an inverse mapping argument introduced in [8]. For this, we consider the following linearized system:

$$
\begin{cases}z_{t}-\Delta z+(\bar{y} \cdot \nabla) z+(z \cdot \nabla) \bar{y}+\nabla q=f_{1}+\rho e_{N}+v_{1} 1_{\omega} & \text { in } Q, \\ \rho_{t}-\Delta \rho+\bar{y} \cdot \nabla \rho+\nabla \cdot(\bar{\theta} z)=f_{2}+v_{2} 1_{\omega} & \text { in } Q, \\ \nabla \cdot z=0 & \text { in } Q, \\ z=0, \quad \rho=0 & \text { on } \Sigma, \\ z(0)=z^{0}, \quad \rho(0)=\rho^{0} & \text { in } \Omega,\end{cases}
$$

where $f_{1}$ and $f_{2}$ are appropriate functions decaying exponentially when $t \rightarrow T^{-}$(see Proposition 2 below).

The goal will be to prove the null controllability for this system, say, we will find two controls $v_{1}$ and $v_{2}$ such that (12) holds for every (regular enough) initial condition $\left(z^{0}, \rho^{0}\right)$. Then, the previous commented argument will provide the desired local result.

The most important tool to prove the null controllability of the linear system (13) is a global Carleman inequality for the solutions of its adjoint system, that is to say,

$$
\begin{cases}-\varphi_{t}-\Delta \varphi-D \varphi \bar{y}+\nabla \pi=g_{1}+\bar{\theta} \nabla \psi & \text { in } Q, \\ -\psi_{t}-\Delta \psi-\bar{y} \cdot \nabla \psi=g_{2}+\varphi \cdot e_{N} & \text { in } Q, \\ \nabla \cdot \varphi=0 & \text { in } Q, \\ \varphi=0, \quad \psi=0 & \text { on } \Sigma, \\ \varphi(T)=\varphi^{0}, \quad \psi(T)=\psi^{0} & \text { in } \Omega .\end{cases}
$$

Here, $D \varphi$ stands for the simetrized gradient

$$
D \varphi=\nabla \varphi+\nabla \varphi^{t} .
$$

In fact, this inequality will contain global terms with the $L^{2}$-weighted norm of $\varphi$ and $\psi$ in the left-hand side, while local terms of $\varphi$ and $\psi$ and global integrals of $g_{1}$ and $g_{2}$ will appear in its right-hand side. We present now a schema of the proof of this estimate:

FIRST PART: First, we apply the Carleman inequality for the heat equation with right-hand sides in $L^{2}(Q)$ and Dirichlet boundary conditions (see [4]) to both the equation satisfied by $\varphi_{i}(1 \leqslant i \leqslant N)$ and the one satisfied by $\psi$. Here, we are viewing the terms $\left(D \varphi \bar{y}-\nabla \pi+g_{1}+\bar{\theta} \nabla \psi\right)_{i}$ and $\bar{y} \cdot \nabla \psi+g_{2}+\varphi \cdot e_{N}$ as right-hand sides. After some arrangements, we get an inequality of the form

$$
I_{1}(\varphi, \psi) \leqslant C\left(\iint_{Q} \rho_{1}^{2}\left(\left|g_{1}\right|^{2}+\left|g_{2}\right|^{2}+|\nabla \pi|^{2}\right) \mathrm{d} x \mathrm{~d} t+\iint_{\omega \times(0, T)} \tilde{\rho}_{1}^{2}\left(|\varphi|^{2}+|\psi|^{2}\right) \mathrm{d} x \mathrm{~d} t\right) .
$$

Here, $I_{1}$ contains several global integrals of $\varphi$ and $\psi$ (see inequality (22)).

SECOND PART: Then, we localize the global term of the pressure using the arguments of [9]. This leads to the estimate

$$
I_{1}(\varphi, \psi) \leqslant C\left(\iint_{Q} \rho_{2}^{2}\left(\left|g_{1}\right|^{2}+\left|g_{2}\right|^{2}\right) \mathrm{d} x \mathrm{~d} t+\iint_{\omega \times(0, T)}\left(\tilde{\rho}_{2}^{2}\left(|\varphi|^{2}+|\psi|^{2}\right)+\bar{\rho}_{2}^{2}|\pi|^{2}\right) \mathrm{d} x \mathrm{~d} t\right)
$$

(see inequality (30)). 
THIRD PART: In the last stage, we estimate the local term of the pressure in terms of $\varepsilon I_{1}(\varphi, \psi)$, global terms of $\left|g_{1}\right|^{2}+\left|g_{2}\right|^{2}$ and local terms of $|\varphi|^{2}+|\psi|^{2}$. The techniques employed here are similar to those of [3]. The conclusion is the desired Carleman estimate (17), which is stated in Proposition 1 below.

The paper is organized as follows: we prove the Carleman inequality for system (14) in Section 2. Section 3 deals with the null controllability result for the linear control system with a right-hand side (13). The proof of the null controllability result for system (11) is given in Section 4. Finally, we include a technical result needed for the proof of the Carleman inequality in an Appendix.

\section{Carleman inequality for the adjoint system}

Let us consider the adjoint system (14) (defined in the introduction), where $g_{1}$ and $g_{2}$ are $L^{2}$ functions.

In this section, we will obtain a suitable Carleman inequality for this system. This will provide a null controllability result for the linear system (13) for suitable $f_{1}$ and $f_{2}$ (Section 3 ).

Before stating this result, let us introduce several weight functions which will be useful in the sequel. For certain positive numbers $s$ and $\lambda$, we set

$$
\begin{aligned}
& \alpha(x, t)=\frac{\mathrm{e}^{(5 / 4) \lambda m\left\|\eta^{0}\right\|_{\infty}}-\mathrm{e}^{\lambda\left(m\left\|\eta^{0}\right\|_{\infty}+\eta^{0}(x)\right)}}{t^{4}(T-t)^{4}}, \\
& \xi(x, t)=\frac{\mathrm{e}^{\lambda\left(m\left\|\eta^{0}\right\|_{\infty}+\eta^{0}(x)\right)}}{t^{4}(T-t)^{4}}, \\
& \alpha^{*}(t)=\max _{x \in \bar{\Omega}} \alpha(x, t), \quad \xi^{*}(t)=\min _{x \in \bar{\Omega}} \xi(x, t), \\
& \hat{\alpha}(t)=\min _{x \in \bar{\Omega}} \alpha(x, t), \quad \hat{\xi}(t)=\max _{x \in \bar{\Omega}} \xi(x, t),
\end{aligned}
$$

where $m>4$ is a fixed real number and $\eta^{0} \in C^{2}(\bar{\Omega})$ is a function that verifies

$$
\eta^{0}>0 \quad \text { in } \Omega, \quad \eta^{0} \equiv 0 \quad \text { on } \partial \Omega, \quad\left|\nabla \eta^{0}\right|>0 \quad \text { in } \Omega \backslash \bar{\omega}_{1} .
$$

Here, $\omega_{1}$ is an open subset of $\omega$. We remark that these weights functions were already used in [3] in order to obtain a Carleman estimate for the Stokes system.

Let us also introduce the following notation:

$$
\begin{aligned}
I(s, \lambda ; \varphi)= & s^{-1} \iint_{Q} \mathrm{e}^{-2 s \alpha} \xi^{-1}\left|\varphi_{t}\right|^{2} \mathrm{~d} x \mathrm{~d} t+s^{-1} \iint_{Q} \mathrm{e}^{-2 s \alpha} \xi^{-1}|\Delta \varphi|^{2} \mathrm{~d} x \mathrm{~d} t \\
& +s \lambda^{2} \iint_{Q} \mathrm{e}^{-2 s \alpha} \xi|\nabla \varphi|^{2} \mathrm{~d} x \mathrm{~d} t+s^{3} \lambda^{4} \iint_{Q} \mathrm{e}^{-2 s \alpha} \xi^{3}|\varphi|^{2} \mathrm{~d} x \mathrm{~d} t .
\end{aligned}
$$

Then, we have:

Proposition 1. Let us assume that $(\bar{y}, \bar{\theta})$ satisfy (5) and $g_{1, i}, g_{2} \in L^{2}(Q)(1 \leqslant i \leqslant N)$. Then, there exist three positive constants $\widehat{C}, \hat{s}$ and $\hat{\lambda}$ just depending on $\Omega$ and $\omega$ such that, for every $\varphi^{0} \in H$ and every $\psi^{0} \in L^{2}(\Omega)$, we have

$$
\begin{aligned}
I(s, \lambda ; \varphi)+I(s, \lambda ; \psi) \leqslant & \widehat{C}\left(1+T^{2}\right)\left(s^{16} \lambda^{48} \iint_{\omega \times(0, T)} \mathrm{e}^{-8 s \hat{\alpha}+6 s \alpha^{*}} \hat{\xi}^{16}\left(|\varphi|^{2}+|\psi|^{2}\right) \mathrm{d} x \mathrm{~d} t\right. \\
& \left.+s^{15 / 2} \lambda^{24} \iint_{Q} \mathrm{e}^{-4 s \hat{\alpha}+2 s \alpha^{*}} \hat{\xi}^{15 / 2}\left(\left|g_{1}\right|^{2}+\left|g_{2}\right|^{2}\right) \mathrm{d} x \mathrm{~d} t\right)
\end{aligned}
$$


for $s \geqslant \hat{s}\left(T^{4}+T^{8}\right)$ and

$$
\lambda \geqslant \hat{\lambda}\left(1+\|\bar{y}\|_{\infty}+\|\bar{\theta}\|_{\infty}+\left\|\bar{y}_{t}\right\|_{L^{2}\left(0, T ; L^{r}(\Omega)^{N}\right)}^{2}+\left\|\bar{\theta}_{t}\right\|_{L^{2}\left(0, T ; L^{r}(\Omega)\right)}^{2}+\mathrm{e}^{\hat{\lambda} T\left(1+\|\bar{y}\|_{\infty}^{2}+\|\bar{\theta}\|_{\infty}^{2}\right)}\right) .
$$

We are going to present this estimate in two subsections. In the first one, we will concentrate in the localization of the pressure $\pi$. Then, we will eliminate it using the terms in the left-hand side of our inequality.

All along this proof, $C$ will denote a generic positive constant that can eventually depend on $\Omega$ and $\omega$ and that may vary from one line to the next one.

\subsection{Localization of the pressure}

Let us first apply the Carleman inequality for the heat system with right-hand side in $L^{2}(Q)$ to each component of $\varphi$. See [4] for the proof and for the explicit dependence with respect to $s, \lambda$ and $T$ see for instance [2]. We get

$$
\begin{aligned}
I(s, \lambda ; \varphi) \leqslant & C\left(\|\bar{y}\|_{\infty}^{2} \iint_{Q} \mathrm{e}^{-2 s \alpha}|\nabla \varphi|^{2} \mathrm{~d} x \mathrm{~d} t+\|\bar{\theta}\|_{\infty}^{2} \iint_{Q} \mathrm{e}^{-2 s \alpha}|\nabla \psi|^{2} \mathrm{~d} x \mathrm{~d} t+\iint_{Q} \mathrm{e}^{-2 s \alpha}|\nabla \pi|^{2} \mathrm{~d} x \mathrm{~d} t\right. \\
& \left.+s^{3} \lambda^{4} \iint_{\omega_{1} \times(0, T)} \mathrm{e}^{-2 s \alpha} \xi^{3}|\varphi|^{2} \mathrm{~d} x \mathrm{~d} t+\iint_{Q} \mathrm{e}^{-2 s \alpha}\left|g_{1}\right|^{2} \mathrm{~d} x \mathrm{~d} t\right),
\end{aligned}
$$

for $s \geqslant C\left(T^{7}+T^{8}\right)$ and $\lambda \geqslant C$. We remark here that, thanks to the definition of $\xi$ (see (16)), we have

$$
\xi^{-1} \leqslant 2^{-8} T^{8}
$$

Now, we are able to eliminate the first term in the right-hand side of (18) with the term in $s \lambda^{2}$ that appears in the expression of $I(s, \lambda ; \varphi)$, if we take $\lambda$ to be large enough. This way, we find

$$
\begin{aligned}
I(s, \lambda ; \varphi) \leqslant & C\left(\|\bar{\theta}\|_{\infty}^{2} \iint_{Q} \mathrm{e}^{-2 s \alpha}|\nabla \psi|^{2} \mathrm{~d} x \mathrm{~d} t+\iint_{Q} \mathrm{e}^{-2 s \alpha}|\nabla \pi|^{2} \mathrm{~d} x \mathrm{~d} t\right. \\
& \left.+s^{3} \lambda^{4} \iint_{\omega_{1} \times(0, T)} \mathrm{e}^{-2 s \alpha} \xi^{3}|\varphi|^{2} \mathrm{~d} x \mathrm{~d} t+\iint_{Q} \mathrm{e}^{-2 s \alpha}\left|g_{1}\right|^{2} \mathrm{~d} x \mathrm{~d} t\right),
\end{aligned}
$$

for $s \geqslant C\left(T^{7}+T^{8}\right)$ and $\lambda \geqslant C\left(1+\|\bar{y}\|_{\infty}\right)$.

Applying again the Carleman estimate to the heat equation fulfilled by $\psi$, we find

$$
\begin{aligned}
I(s, \lambda ; \psi) \leqslant & C\left(\|\bar{y}\|_{\infty}^{2} \iint_{Q} \mathrm{e}^{-2 s \alpha}|\nabla \psi|^{2} \mathrm{~d} x \mathrm{~d} t+\iint_{Q} \mathrm{e}^{-2 s \alpha}\left|\varphi_{N}\right|^{2} \mathrm{~d} x \mathrm{~d} t\right. \\
& \left.+s^{3} \lambda^{4} \iint_{\omega_{1} \times(0, T)} \mathrm{e}^{-2 s \alpha} \xi^{3}|\psi|^{2} \mathrm{~d} x \mathrm{~d} t+\iint_{Q} \mathrm{e}^{-2 s \alpha}\left|g_{2}\right|^{2} \mathrm{~d} x \mathrm{~d} t\right),
\end{aligned}
$$

for $s \geqslant C\left(T^{7}+T^{8}\right)$ and $\lambda \geqslant C$, where we have denoted $\varphi_{N}=\varphi \cdot e_{N}$. The same argument used above yields

$$
I(s, \lambda ; \psi) \leqslant C\left(\iint_{Q} \mathrm{e}^{-2 s \alpha}\left|\varphi_{N}\right|^{2} \mathrm{~d} x \mathrm{~d} t+s^{3} \lambda^{4} \iint_{\omega_{1} \times(0, T)} \mathrm{e}^{-2 s \alpha} \xi^{3}|\psi|^{2} \mathrm{~d} x \mathrm{~d} t+\iint_{Q} \mathrm{e}^{-2 s \alpha}\left|g_{2}\right|^{2} \mathrm{~d} x \mathrm{~d} t\right),
$$

for any $s \geqslant C\left(T^{7}+T^{8}\right)$ and any $\lambda \geqslant C\left(1+\|\bar{y}\|_{\infty}\right)$. 
The addition of (19) and (20) gives

$$
\begin{aligned}
I(s, \lambda ; \varphi)+I(s, \lambda ; \psi) \leqslant & C\left(\iint_{Q} \mathrm{e}^{-2 s \alpha}\left|\varphi_{N}\right|^{2} \mathrm{~d} x \mathrm{~d} t+\iint_{Q} \mathrm{e}^{-2 s \alpha}|\nabla \pi|^{2} \mathrm{~d} x \mathrm{~d} t\right. \\
& +\|\bar{\theta}\|_{\infty}^{2} \iint_{Q} \mathrm{e}^{-2 s \alpha}|\nabla \psi|^{2} \mathrm{~d} x \mathrm{~d} t+s^{3} \lambda^{4} \iint_{\omega_{1} \times(0, T)} \mathrm{e}^{-2 s \alpha} \xi^{3}|\varphi|^{2} \mathrm{~d} x \mathrm{~d} t \\
& \left.+s^{3} \lambda^{4} \iint_{\omega_{1} \times(0, T)} \mathrm{e}^{-2 s \alpha} \xi^{3}|\psi|^{2} \mathrm{~d} x \mathrm{~d} t+\iint_{Q} \mathrm{e}^{-2 s \alpha}\left(\left|g_{1}\right|^{2}+\left|g_{2}\right|^{2}\right) \mathrm{d} x \mathrm{~d} t\right),
\end{aligned}
$$

for any $s \geqslant C\left(T^{7}+T^{8}\right)$ and any $\lambda \geqslant C\left(1+\|\bar{y}\|_{\infty}\right)$.

Several computations similar to the previous ones lead to the absorption of the first and the third terms in the right-hand side of (21), making use of the terms in $s^{3} \lambda^{4}$ and in $s \lambda^{2}$ appearing in $I(s, \lambda ; \varphi)$ and $I(s, \lambda ; \psi)$, respectively. This is possible with a choice like $s \geqslant C T^{8}$ and $\lambda \geqslant C\left(1+\|\bar{\theta}\|_{\infty}\right)$. Therefore, we have

$$
\begin{aligned}
I(s, \lambda ; \varphi)+I(s, \lambda ; \psi) \leqslant & C\left(\iint_{Q} \mathrm{e}^{-2 s \alpha}|\nabla \pi|^{2} \mathrm{~d} x \mathrm{~d} t+s^{3} \lambda^{4} \iint_{\omega_{1} \times(0, T)} \mathrm{e}^{-2 s \alpha} \xi^{3}|\varphi|^{2} \mathrm{~d} x \mathrm{~d} t\right. \\
& \left.+s^{3} \lambda^{4} \iint_{\omega_{1} \times(0, T)} \mathrm{e}^{-2 s \alpha} \xi^{3}|\psi|^{2} \mathrm{~d} x \mathrm{~d} t+\iint_{Q} \mathrm{e}^{-2 s \alpha}\left(\left|g_{1}\right|^{2}+\left|g_{2}\right|^{2}\right) \mathrm{d} x \mathrm{~d} t\right),
\end{aligned}
$$

for $s \geqslant C\left(T^{7}+T^{8}\right)$ and $\lambda \geqslant C\left(1+\|\bar{y}\|_{\infty}+\|\bar{\theta}\|_{\infty}\right)$.

So far, the arguments used are classical. The important part of this paragraph starts now, where we are going to localize the global term of the presssure in (22). To this end, we employ similar arguments to those developed in [3].

Let us then look at the (weak) equation satisfied by the pressure, which can be found taking the divergence operator in the movement equation of (14):

$$
\Delta \pi(t)=\nabla \cdot\left(D \varphi(t) \bar{y}(t)+\bar{\theta}(t) \nabla \psi(t)+g_{1}(t)\right) \quad \text { in } \Omega \text {, a.e. } t \in(0, T) .
$$

Watching the right-hand side of (23) like a $H^{-1}$ term, we apply the main result in [9], say, a Carleman estimate for the weak solutions of elliptic equations. This gives the existence of two constants $\bar{\sigma}>1$ and $\bar{\lambda}>1$, such that

$$
\begin{aligned}
& \int_{\Omega} \mathrm{e}^{2 \sigma \eta_{1}}|\nabla \pi(t)|^{2} \mathrm{~d} x+\sigma^{2} \lambda^{2} \int_{\Omega} \mathrm{e}^{2 \sigma \eta_{1}} \eta_{1}^{2}|\pi(t)|^{2} \mathrm{~d} x \\
& \leqslant C\left(\sigma \int_{\Omega} \mathrm{e}^{2 \sigma \eta_{1}} \eta_{1}\left|D \varphi(t) \bar{y}(t)+\bar{\theta}(t) \nabla \psi(t)+g_{1}(t)\right|^{2} \mathrm{~d} x+\sigma^{1 / 2} \mathrm{e}^{2 \sigma}\|\pi(t)\|_{H^{1 / 2}(\partial \Omega)}^{2}\right. \\
& \left.\quad+\int_{\omega_{1}} \mathrm{e}^{2 \sigma \eta_{1}}|\nabla \pi(t)|^{2} \mathrm{~d} x+\sigma^{2} \lambda^{2} \int_{\omega_{1}} \mathrm{e}^{2 \sigma \eta_{1}} \eta_{1}^{2}|\pi(t)|^{2} \mathrm{~d} x\right)
\end{aligned}
$$

for any $\sigma \geqslant \bar{\sigma}$ and any $\lambda \geqslant \bar{\lambda}$. Here, we have denoted for each $\lambda>0$,

$$
\eta_{1}(x)=\mathrm{e}^{\lambda \eta^{0}(x)}, \quad x \in \Omega .
$$

The next step will be now to eliminate the local term in $\nabla \pi$. For this, we consider an open set $\omega_{2}$ such that $\omega_{1} \Subset \omega_{2} \Subset \omega$ and we define $\zeta \in C^{2}(\Omega)$ such that

$$
\operatorname{supp} \zeta \subset \omega_{2}, \quad \zeta \equiv 1 \quad \text { in } \omega_{1}, \quad 0 \leqslant \zeta \leqslant 1 .
$$


Let us integrate by parts several times:

$$
\begin{aligned}
\int_{\omega_{1}} \mathrm{e}^{2 \sigma \eta_{1}}|\nabla \pi(t)|^{2} \mathrm{~d} x \leqslant & \int_{\omega_{2}} \mathrm{e}^{2 \sigma \eta_{1}} \zeta|\nabla \pi(t)|^{2} \mathrm{~d} x \\
= & -\int_{\omega_{2}} \mathrm{e}^{2 \sigma \eta_{1}} \zeta \Delta \pi(t) \pi(t) \mathrm{d} x-\int_{\omega_{2}} \mathrm{e}^{2 \sigma \eta_{1}}(\nabla \zeta \cdot \nabla \pi(t)) \pi(t) \mathrm{d} x \\
& -2 \sigma \lambda \int_{\omega_{2}} \mathrm{e}^{2 \sigma \eta_{1}} \eta_{1} \zeta\left(\nabla \eta^{0} \cdot \nabla \pi(t)\right) \pi(t) \mathrm{d} x .
\end{aligned}
$$

From (23) and new integrations by parts, we get the following for the first term:

$$
\begin{aligned}
\int_{\omega_{2}} \mathrm{e}^{2 \sigma \eta_{1}} \zeta \Delta \pi(t) \pi(t) \mathrm{d} x= & \int_{\omega_{2}} \mathrm{e}^{2 \sigma \eta_{1}} \zeta \nabla \cdot\left(D \varphi(t) \bar{y}(t)+\bar{\theta}(t) \nabla \psi(t)+g_{1}(t)\right) \pi(t) \mathrm{d} x \\
= & -\int_{\omega_{2}} \mathrm{e}^{2 \sigma \eta_{1}} \nabla \zeta \cdot\left(D \varphi(t) \bar{y}(t)+\bar{\theta}(t) \nabla \psi(t)+g_{1}(t)\right) \pi(t) \mathrm{d} x \\
& -2 \sigma \lambda \int_{\omega_{2}} \mathrm{e}^{2 \sigma \eta_{1}} \eta_{1} \zeta \nabla \eta^{0} \cdot\left(D \varphi(t) \bar{y}(t)+\bar{\theta}(t) \nabla \psi(t)+g_{1}(t)\right) \pi(t) \mathrm{d} x \\
& -\int_{\omega_{2}} \mathrm{e}^{2 \sigma \eta_{1}} \zeta\left(D \varphi(t) \bar{y}(t)+\bar{\theta}(t) \nabla \psi(t)+g_{1}(t)\right) \cdot \nabla \pi(t) \mathrm{d} x .
\end{aligned}
$$

Consequently, for $\sigma, \lambda \geqslant C$, we have

$$
\begin{gathered}
-\int_{\omega_{2}} \mathrm{e}^{2 \sigma \eta_{1}} \zeta \Delta \pi(t) \pi(t) \mathrm{d} x \leqslant C\left(\sigma^{2} \lambda^{2} \int_{\omega_{2}} \mathrm{e}^{2 \sigma \eta_{1}} \eta_{1}^{2}|\pi(t)|^{2} \mathrm{~d} x+\|\bar{y}\|_{\infty}^{2} \int_{\omega_{2}} \mathrm{e}^{2 \sigma \eta_{1}}|\nabla \varphi(t)|^{2} \mathrm{~d} x\right. \\
\left.\quad+\|\bar{\theta}\|_{\infty}^{2} \int_{\omega_{2}} \mathrm{e}^{2 \sigma \eta_{1}}|\nabla \psi(t)|^{2} \mathrm{~d} x+\int_{\omega_{2}} \mathrm{e}^{2 \sigma \eta_{1}}\left|g_{1}(t)\right|^{2} \mathrm{~d} x\right)+\frac{1}{2} \int_{\omega_{2}} \mathrm{e}^{2 \sigma \eta_{1}} \zeta|\nabla \pi(t)|^{2} r \mathrm{~d} x .
\end{gathered}
$$

Analogous computations for the second and the third terms in (25), give

$$
\begin{aligned}
& -\int_{\omega_{2}} \mathrm{e}^{2 \sigma \eta_{1}}(\nabla \zeta \cdot \nabla \pi(t)) \pi(t) \mathrm{d} x-2 \sigma \lambda \int_{\omega_{2}} \mathrm{e}^{2 \sigma \eta_{1}} \eta_{1} \zeta\left(\nabla \eta^{0} \cdot \nabla \pi(t)\right) \pi(t) \mathrm{d} x \\
& \quad \leqslant C \sigma^{2} \lambda^{2} \int_{\omega_{2}} \mathrm{e}^{2 \sigma \eta_{1}} \eta_{1}^{2}|\pi(t)|^{2} \mathrm{~d} x
\end{aligned}
$$

for $\sigma, \lambda \geqslant C$.

Combining (26) and (27) with (25), we obtain

$$
\begin{aligned}
\int_{\omega_{1}} \mathrm{e}^{2 \sigma \eta_{1}}|\nabla \pi(t)|^{2} \mathrm{~d} x \leqslant & C\left(\sigma^{2} \lambda^{2} \int_{\omega_{2}} \mathrm{e}^{2 \sigma \eta_{1}} \eta_{1}^{2}|\pi(t)|^{2} \mathrm{~d} x+\|\bar{y}\|_{\infty}^{2} \int_{\omega_{2}} \mathrm{e}^{2 \sigma \eta_{1}}|\nabla \varphi(t)|^{2} \mathrm{~d} x\right. \\
& \left.+\|\bar{\theta}\|_{\infty}^{2} \int_{\omega_{2}} \mathrm{e}^{2 \sigma \eta_{1}}|\nabla \psi(t)|^{2} \mathrm{~d} x+\int_{\omega_{2}} \mathrm{e}^{2 \sigma \eta_{1}}\left|g_{1}(t)\right|^{2} \mathrm{~d} x\right)
\end{aligned}
$$

for $\sigma, \lambda \geqslant C(\Omega, \omega)$, which together with (24) yields 


$$
\begin{aligned}
& \int_{\Omega} \mathrm{e}^{2 \sigma \eta_{1}}|\nabla \pi(t)|^{2} \mathrm{~d} x+\sigma^{2} \lambda^{2} \int_{\Omega} \mathrm{e}^{2 \sigma \eta_{1}} \eta_{1}^{2}|\pi(t)|^{2} \mathrm{~d} x \\
& \leqslant C\left(\sigma\|\bar{y}\|_{\infty}^{2} \int_{\Omega} \mathrm{e}^{2 \sigma \eta_{1}} \eta_{1}|\nabla \varphi(t)|^{2} \mathrm{~d} x+\sigma\|\bar{\theta}\|_{\infty}^{2} \int_{\Omega} \mathrm{e}^{2 \sigma \eta_{1}} \eta_{1}|\nabla \psi(t)|^{2} \mathrm{~d} x+\sigma \int_{\Omega} \mathrm{e}^{2 \sigma \eta_{1}} \eta_{1}\left|g_{1}(t)\right|^{2} \mathrm{~d} x\right. \\
& \left.\quad+\sigma^{1 / 2} \mathrm{e}^{2 \sigma}\|\pi(t)\|_{H^{1 / 2}(\partial \Omega)}^{2}+\sigma^{2} \lambda^{2} \int_{\omega_{2}} \mathrm{e}^{2 \sigma \eta_{1}} \eta_{1}^{2}|\pi(t)|^{2} \mathrm{~d} x\right)
\end{aligned}
$$

for $\sigma, \lambda \geqslant C$ and for almost every $t \in(0, T)$.

The next step will be to connect this inequality with (22). For this, let us set

$$
\sigma=\frac{s}{t^{4}(T-t)^{4}} \mathrm{e}^{\lambda m\left\|\eta^{0}\right\|_{\infty}}
$$

let us multiply the expression (28) by

$$
\exp \left\{-2 s \frac{\mathrm{e}^{(5 / 4) \lambda m\left\|\eta^{0}\right\|_{\infty}}}{t^{4}(T-t)^{4}}\right\}
$$

and then integrate it between $t=0$ and $t=T$. This provides

$$
\begin{aligned}
& \iint_{Q} \mathrm{e}^{-2 s \alpha}|\nabla \pi|^{2} \mathrm{~d} x \mathrm{~d} t+s^{2} \lambda^{2} \iint_{Q} \mathrm{e}^{-2 s \alpha} \xi^{2}|\pi|^{2} \mathrm{~d} x \mathrm{~d} t \\
& \leqslant C\left(s\|\bar{y}\|_{\infty}^{2} \iint_{Q} \mathrm{e}^{-2 s \alpha} \xi|\nabla \varphi|^{2} \mathrm{~d} x \mathrm{~d} t+s\|\bar{\theta}\|_{\infty}^{2} \iint_{Q} \mathrm{e}^{-2 s \alpha} \xi|\nabla \psi|^{2} \mathrm{~d} x \mathrm{~d} t+s \iint_{Q} \mathrm{e}^{-2 s \alpha} \xi\left|g_{1}\right|^{2} \mathrm{~d} x \mathrm{~d} t\right. \\
& \left.\quad+s^{1 / 2} \int_{0}^{T} \mathrm{e}^{-2 s \alpha^{*}} \xi^{* 1 / 2}\|\pi(t)\|_{H^{1 / 2}(\partial \Omega)}^{2} \mathrm{~d} t+s^{2} \lambda^{2} \iint_{\omega_{2} \times(0, T)} \mathrm{e}^{-2 s \alpha} \xi^{2}|\pi|^{2} \mathrm{~d} x \mathrm{~d} t\right)
\end{aligned}
$$

for any $\lambda \geqslant C$ and any $s \geqslant C T^{8} \mathrm{e}^{-\lambda m\left\|\eta^{0}\right\|_{\infty}}$. Plugging this inequality into (22), we find

$$
\begin{aligned}
I(s, \lambda ; \varphi)+I(s, \lambda ; \psi) \leqslant & C\left(s\|\bar{y}\|_{\infty}^{2} \iint_{Q} \mathrm{e}^{-2 s \alpha} \xi|\nabla \varphi|^{2} \mathrm{~d} x \mathrm{~d} t\right. \\
& +s\|\bar{\theta}\|_{\infty}^{2} \iint_{Q} \mathrm{e}^{-2 s \alpha} \xi|\nabla \psi|^{2} \mathrm{~d} x \mathrm{~d} t+s^{1 / 2} \int_{0}^{T} \mathrm{e}^{-2 s \alpha^{*} \xi^{* 1 / 2}}(t)\|\pi(t)\|_{H^{1 / 2}(\partial \Omega)}^{2} \mathrm{~d} t \\
& +s^{2} \lambda^{2} \iint_{\omega_{2} \times(0, T)} \mathrm{e}^{-2 s \alpha} \xi^{2}|\pi|^{2} \mathrm{~d} x \mathrm{~d} t+s^{3} \lambda^{4} \iint_{\omega_{1} \times(0, T)} \mathrm{e}^{-2 s \alpha} \xi^{3}|\varphi|^{2} \mathrm{~d} x \mathrm{~d} t \\
& +s^{3} \lambda^{4} \iint_{\omega_{1} \times(0, T)} \mathrm{e}^{-2 s \alpha} \xi^{3}|\psi|^{2} \mathrm{~d} x \mathrm{~d} t+s \iint_{Q} \mathrm{e}^{-2 s \alpha} \xi\left|g_{1}\right|^{2} \mathrm{~d} x \mathrm{~d} t \\
& \left.+\iint_{Q} \mathrm{e}^{-2 s \alpha}\left|g_{2}\right|^{2} \mathrm{~d} x \mathrm{~d} t\right)
\end{aligned}
$$


for any $s \geqslant C\left(T^{7}+T^{8}\right)$ and any $\lambda \geqslant C\left(1+\|\bar{y}\|_{\infty}+\|\bar{\theta}\|_{\infty}\right)$. Similarly as we did above, it follows directly from a choice like $s \geqslant C T^{8}$ and $\lambda \geqslant C\left(\|\bar{y}\|_{\infty}+\|\bar{\theta}\|_{\infty}\right)$, that the two first terms in the right-hand side of the last inequality can be absorbed.

Consequently, we have

$$
\begin{aligned}
I(s, \lambda ; \varphi)+I(s, \lambda ; \psi) \leqslant & C\left(s^{1 / 2} \int_{0}^{T} \mathrm{e}^{-2 s \alpha^{*}} \xi^{* 1 / 2}\|\pi(t)\|_{H^{1 / 2}(\partial \Omega)}^{2} \mathrm{~d} t+s^{2} \lambda^{2} \iint_{\omega_{2} \times(0, T)} \mathrm{e}^{-2 s \alpha} \xi^{2}|\pi|^{2} \mathrm{~d} x \mathrm{~d} t\right. \\
& +s^{3} \lambda^{4} \iint_{\omega_{1} \times(0, T)} \mathrm{e}^{-2 s \alpha} \xi^{3}|\varphi|^{2} \mathrm{~d} x \mathrm{~d} t+s^{3} \lambda^{4} \iint_{\omega_{1} \times(0, T)} \mathrm{e}^{-2 s \alpha} \xi^{3}|\psi|^{2} \mathrm{~d} x \mathrm{~d} t \\
& \left.+s \iint_{Q} \mathrm{e}^{-2 s \alpha} \xi\left|g_{1}\right|^{2} \mathrm{~d} x \mathrm{~d} t+\iint_{Q} \mathrm{e}^{-2 s \alpha}\left|g_{2}\right|^{2} \mathrm{~d} x \mathrm{~d} t\right)
\end{aligned}
$$

for any $s \geqslant C\left(T^{7}+T^{8}\right)$ and any $\lambda \geqslant C\left(1+\|\bar{y}\|_{\infty}+\|\bar{\theta}\|_{\infty}\right)$.

Now, we will eliminate the term of the trace of the pressure. The tools we use here are classical regularity estimates for the solutions of Stokes systems. More precisely, let us introduce for

$$
\beta=s^{1 / 4} \mathrm{e}^{-s \alpha^{*}} \xi^{* 1 / 4},
$$

the functions

$$
\hat{\varphi}=\beta \varphi, \quad \hat{\pi}=\beta \pi
$$

and let us see which system they fulfill:

$$
\begin{cases}-\hat{\varphi}_{t}-\Delta \hat{\varphi}+\nabla \hat{\pi}=\beta D \varphi \bar{y}+\beta \bar{\theta} \nabla \psi+\beta g_{1}-\beta_{t} \varphi & \text { in } Q, \\ \nabla \cdot \hat{\varphi}=0 & \text { in } Q, \\ \hat{\varphi}=0 & \text { on } \Sigma, \\ \hat{\varphi}(T)=0 & \text { in } \Omega .\end{cases}
$$

Regularity estimates for this system (see, for instance, [11]) give

$$
\|\hat{\pi}\|_{L^{2}\left(0, T ; H^{1}(\Omega)\right)} \leqslant C\left(\|\beta D \varphi \bar{y}\|_{L^{2}(Q)^{N}}+\|\beta \bar{\theta} \nabla \psi\|_{L^{2}(Q)^{N}}+\left\|\beta g_{1}\right\|_{L^{2}(Q)^{N}}+\left\|\beta_{t} \varphi\right\|_{L^{2}(Q)^{N}}\right),
$$

for a positive constant $C=C(\Omega)$. From the definition of $\xi^{*}$ and $\alpha^{*}$ (see (16)), we deduce

$$
\beta_{t}=s^{1 / 4} \mathrm{e}^{-s \alpha^{*}}\left(\frac{1}{4} \xi^{*-3 / 4}\left(\xi^{*}\right)_{t}-s\left(\alpha^{*}\right)_{t} \xi^{* 1 / 4}\right) \leqslant C s^{1 / 4} T \mathrm{e}^{-s \alpha}\left(\xi^{1 / 2}+s \xi^{3 / 2}\right) \leqslant C s^{5 / 4} T \mathrm{e}^{-s \alpha} \xi^{3 / 2}
$$

for $s \geqslant C T^{8}$. Therefore, using the continuity of the trace operator, we have

$$
\begin{aligned}
\int_{0}^{T}\|\hat{\pi}(t)\|_{H^{1 / 2}(\partial \Omega)}^{2} \mathrm{~d} t \leqslant & C\left(s^{1 / 2}\|\bar{y}\|_{\infty}^{2} \iint_{Q} \mathrm{e}^{-2 s \alpha} \xi^{1 / 2}|\nabla \varphi|^{2} \mathrm{~d} x \mathrm{~d} t+s^{1 / 2}\|\bar{\theta}\|_{\infty}^{2} \iint_{Q} \mathrm{e}^{-2 s \alpha} \xi^{1 / 2}|\nabla \psi|^{2} \mathrm{~d} x \mathrm{~d} t\right. \\
& \left.+s^{1 / 2} \iint_{Q} \mathrm{e}^{-2 s \alpha} \xi^{1 / 2}\left|g_{1}\right|^{2} \mathrm{~d} x \mathrm{~d} t+s^{5 / 2} T^{2} \iint_{Q} \mathrm{e}^{-2 s \alpha} \xi^{3}|\varphi|^{2} \mathrm{~d} x \mathrm{~d} t\right)
\end{aligned}
$$

for $s \geqslant C T^{8}$. Let us now connect this estimate of the trace of the pressure with (29). We get 


$$
\begin{aligned}
& I(s, \lambda ; \varphi)+I(s, \lambda ; \psi) \leqslant C\left(s^{2} \lambda^{2} \iint_{\omega_{2} \times(0, T)} \mathrm{e}^{-2 s \alpha} \xi^{2}|\pi|^{2} \mathrm{~d} x \mathrm{~d} t+s^{3} \lambda^{4} \iint_{\omega_{1} \times(0, T)} \mathrm{e}^{-2 s \alpha} \xi^{3}|\varphi|^{2} \mathrm{~d} x \mathrm{~d} t\right. \\
& \left.\quad+s^{3} \lambda^{4} \iint_{\omega_{1} \times(0, T)} \mathrm{e}^{-2 s \alpha} \xi^{3}|\psi|^{2} \mathrm{~d} x \mathrm{~d} t+s \iint_{Q} \mathrm{e}^{-2 s \alpha} \xi\left|g_{1}\right|^{2} \mathrm{~d} x \mathrm{~d} t+\iint_{Q} \mathrm{e}^{-2 s \alpha}\left|g_{2}\right|^{2} \mathrm{~d} x \mathrm{~d} t\right),
\end{aligned}
$$

for any $s \geqslant C\left(T^{4}+T^{8}\right)$ and any $\lambda \geqslant C\left(1+\|\bar{y}\|_{\infty}+\|\bar{\theta}\|_{\infty}\right)$.

Remark 2. According to an idea of J.-P. Puel, (30) constitutes an important estimate by itself. In fact, one can prove that (30) leads to the null controllability of the linear system (13) when we are controlling not only with $v_{1}$ and $v_{2}$ but through the divergence condition as well. More precisely, we can prove the existence of three controls $v_{1}, v_{2}$ and $v_{3}$ such that the solution of

$$
\begin{cases}z_{t}-\Delta z+(\bar{y} \cdot \nabla) z+(z \cdot \nabla) \bar{y}+\nabla q=f_{1}+\rho e_{N}+v_{1} 1_{\omega} & \text { in } Q, \\ \rho_{t}-\Delta \rho+\bar{y} \cdot \nabla \rho+\nabla \cdot(\bar{\theta} z)=f_{2}+v_{2} 1_{\omega} & \text { in } Q, \\ \nabla \cdot z=v_{3} 1_{\omega} & \text { in } Q, \\ z=0, \quad \rho=0 & \text { on } \Sigma, \\ z(0)=z^{0}, \quad \rho(0)=\rho^{0} & \text { in } \Omega,\end{cases}
$$

verifies $z(T)=0$ and $\rho(T)=0$ in $\Omega$. By an extension of $\Omega$, this also proves the null controllability of (13) with two controls acting on a (little) part of $\partial \Omega$ over the traces of the velocity vector field $z$ and the temperature $\rho$.

\subsection{Local estimate of the pressure}

In this paragraph, we will conclude the proof of inequality (17). For this, we will use similar ideas to those developed in [3].

Indeed, let us choose the pressure $\pi$ to have null mean-value in $\omega_{2}$ :

$$
\int_{\omega_{2}} \pi(t) \mathrm{d} x=0 \quad \text { a.e. } t \in(0, T) .
$$

Then, taking into account the definition of the weight functions given in (16) and Poincare's inequality, we restrict ourselves to the estimate of the term

$$
s^{2} \lambda^{2} \iint_{\omega_{2} \times(0, T)} \mathrm{e}^{-2 s \hat{\alpha}} \hat{\xi}^{2}|\nabla \pi|^{2} \mathrm{~d} x \mathrm{~d} t,
$$

where the weight function $\mathrm{e}^{-2 s \hat{\alpha}} \hat{\xi}^{2}$ does not depend on $x$. Let us remark here that we do not know for the moment any local estimate of the pressure when multiplied by a weight function depending both on $x$ and $t$.

By virtue of the movement equation in (14), we have

$$
\begin{aligned}
& s^{2} \lambda^{2} \iint_{\omega_{2} \times(0, T)} \mathrm{e}^{-2 s \hat{\alpha}} \hat{\xi}^{2}|\nabla \pi|^{2} \mathrm{~d} x \mathrm{~d} t \\
& \quad=s^{2} \lambda^{2} \iint_{\omega_{2} \times(0, T)} \mathrm{e}^{-2 s \hat{\alpha}} \hat{\xi}^{2}\left|\varphi_{t}+\Delta \varphi+D \varphi \bar{y}+\bar{\theta} \nabla \psi+g_{1}\right|^{2} \mathrm{~d} x \mathrm{~d} t .
\end{aligned}
$$

The rest of the proof is intended to estimate the local terms on $\Delta \varphi$ and $\varphi_{t}$. We present now that of $\varphi_{t}$, while the estimate of $\Delta \varphi$ will be given in the Appendix at the end of the paper, since it was already proved in [3] and does not entail a lot of difficulties. 
This proof follows the ideas included in [3], but here we must deal with greater difficulties, as far as a coupled system is involved.

Thus, let us introduce the weight function

$$
\eta(t)=s^{15 / 4} \mathrm{e}^{-2 s \hat{\alpha}+s \alpha^{*}} \hat{\xi}^{15 / 4}
$$

and let $\left(u_{1}, q_{1}, w_{1}\right)$ and $\left(u_{2}, q_{2}, w_{2}\right)$ be the respective solutions to the following systems:

$$
\begin{cases}-u_{1, t}-\Delta u_{1}-D u_{1} \bar{y}+\nabla q_{1}=\eta g_{1}+\bar{\theta} \nabla w_{1} & \text { in } Q, \\ -w_{1, t}-\Delta w_{1}-\bar{y} \cdot \nabla w_{1}=\eta g_{2} & \text { in } Q, \\ \nabla \cdot u_{1}=0 & \text { in } Q, \\ u_{1}=0, \quad w_{1}=0 & \text { on } \Sigma, \\ u_{1}(T)=0, \quad w_{1}(T)=0 & \text { in } \Omega\end{cases}
$$

and

$$
\begin{cases}-u_{2, t}-\Delta u_{2}-D u_{2} \bar{y}+\nabla q_{2}=-\eta^{\prime} \varphi+\bar{\theta} \nabla w_{2} & \text { in } Q, \\ -w_{2, t}-\Delta w_{2}-\bar{y} \cdot \nabla w_{2}=-\eta^{\prime} \psi+\eta \varphi \cdot e_{N} & \text { in } Q, \\ \nabla \cdot u_{2}=0 & \text { in } Q, \\ u_{2}=0, \quad w_{2}=0 & \text { on } \Sigma \\ u_{2}(T)=0, \quad w_{2}(T)=0 & \text { in } \Omega .\end{cases}
$$

It is readily seen that $\left(u_{1}+u_{2}, q_{1}+q_{2}, w_{1}+w_{2}\right)$ solve the same system as $(\eta \varphi, \eta \pi, \eta \psi)$, where $(\varphi, \pi, \psi)$ is the solution to (14). By uniqueness of the Boussinesq system, it must be

$$
\eta \varphi=u_{1}+u_{2}, \quad \eta \pi=q_{1}+q_{2} \quad \text { and } \quad \eta \psi=w_{1}+w_{2} .
$$

Consequently, the term to be bounded is

$$
\begin{aligned}
s^{2} \lambda^{2} \iint_{\omega_{2} \times(0, T)} \mathrm{e}^{-2 s \hat{\alpha}} \hat{\xi}^{2}\left|\varphi_{t}\right|^{2} \mathrm{~d} x \mathrm{~d} t & =s^{2} \lambda^{2} \iint_{\omega_{2} \times(0, T)} \eta^{-2} \mathrm{e}^{-2 s \hat{\alpha}} \hat{\xi}^{2}\left|\eta \varphi_{t}\right|^{2} \mathrm{~d} x \mathrm{~d} t \\
& =s^{-11 / 2} \lambda^{2} \iint_{\omega_{2} \times(0, T)} \mathrm{e}^{-2 s \alpha^{*}+2 s \hat{\alpha}} \hat{\xi}^{-11 / 2}\left|u_{1, t}+u_{2, t}-\eta^{\prime} \varphi\right|^{2} \mathrm{~d} x \mathrm{~d} t,
\end{aligned}
$$

so we will concentrate in estimating the time derivatives of $u_{1}$ and $u_{2}$.

\subsubsection{Estimate of $u_{1, t}$ and $u_{2, t}$}

We first bound the integral of $\mathrm{e}^{-2 s \alpha^{*}+2 s \hat{\alpha}} \hat{\xi}^{-11 / 2}\left|u_{1, t}\right|^{2}$ in $\omega_{2} \times(0, T)$. To do this, we are going to estimate $u_{1, t}$ globally in $\Omega \times(0, T)$ combining regularity estimates for the Stokes and heat systems.

Taking $s$ and $\lambda$ such that $s \geqslant C T^{8}$ and $\lambda \geqslant C$, we get

$$
\mathrm{e}^{-2 s \alpha^{*}+2 s \hat{\alpha}} \leqslant \mathrm{e}^{-C s T^{-8} \mathrm{e}^{\lambda m\left\|\eta \eta^{0}\right\| \infty}} \leqslant \mathrm{e}^{-C \mathrm{e}^{\lambda m\left\|\eta^{0}\right\| \infty}}
$$

and

$$
s^{-11 / 2} \lambda^{2} \hat{\xi}^{-11 / 2} \mathrm{e}^{-2 s \alpha^{*}+2 s \hat{\alpha}} \leqslant C \lambda^{2} \mathrm{e}^{-C \mathrm{e}^{\lambda m\left\|\eta^{0}\right\| \infty}} \leqslant C,
$$

for $\lambda$ large enough.

Combining energy estimates for $u_{1}$ and $w_{1}$, we have

$$
\left\|u_{1}\right\|_{L^{2}(0, T ; V)}^{2}+\left\|w_{1}\right\|_{L^{2}\left(0, T ; H^{1}(\Omega)\right)}^{2} \leqslant C \mathrm{e}^{C T\left(1+\|\bar{y}\|_{\infty}^{2}+\|\bar{\theta}\|_{\infty}^{2}\right)}\left(\left\|\eta g_{1}\right\|_{L^{2}\left(0, T ; H^{-1}(\Omega)^{N}\right)}^{2}+\left\|\eta g_{2}\right\|_{L^{2}\left(0, T ; H^{-1}(\Omega)\right)}^{2}\right) .
$$

Now, we apply regularity estimates to $(33)$ and we deduce that $\left(u_{1}\right)_{i}, w_{1} \in H^{1}\left(0, T ; L^{2}(\Omega)\right) \cap L^{2}\left(0, T ; H^{2}(\Omega)\right)$ and 


$$
\begin{gathered}
\left\|u_{1}\right\|_{H^{1}\left(0, T ; L^{2}(\Omega)^{N}\right)}^{2}+\left\|u_{1}\right\|_{L^{2}\left(0, T ; H^{2}(\Omega)^{N}\right)}^{2}+\left\|w_{1}\right\|_{H^{1}\left(0, T ; L^{2}(\Omega)\right)}^{2}+\left\|w_{1}\right\|_{L^{2}\left(0, T ; H^{2}(\Omega)\right)}^{2} \\
\leqslant C\left(1+\left(\|\bar{y}\|_{\infty}^{2}+\|\bar{\theta}\|_{\infty}^{2}\right) \mathrm{e}^{C T\left(1+\|\bar{y}\|_{\infty}^{2}+\|\bar{\theta}\|_{\infty}^{2}\right)}\right)\left(\left\|\eta g_{1}\right\|_{L^{2}(Q)^{N}}^{2}+\left\|\eta g_{2}\right\|_{L^{2}(Q)}^{2}\right) .
\end{gathered}
$$

Hence, we obtain in particular that

$$
\begin{aligned}
& s^{-11 / 2} \lambda^{2} \iint_{\omega_{2} \times(0, T)} \mathrm{e}^{-2 s \alpha^{*}+2 s \hat{\alpha}} \hat{\xi}^{-11 / 2}\left(\left|u_{1, t}\right|^{2}+\left|\Delta u_{1}\right|^{2}\right) \mathrm{d} x \mathrm{~d} t+\iint_{Q}\left(\left|w_{1, t}\right|^{2}+\left|\Delta w_{1}\right|^{2}\right) \mathrm{d} x \mathrm{~d} t \\
& \leqslant C\left(1+\|\bar{y}\|_{\infty}^{2}+\|\bar{\theta}\|_{\infty}^{2}\right) \mathrm{e}^{C T\left(1+\|\bar{y}\|_{\infty}^{2}+\|\bar{\theta}\|_{\infty}^{2}\right)} \iint_{Q}|\eta|^{2}\left(\left|g_{1}\right|^{2}+\left|g_{2}\right|^{2}\right) \mathrm{d} x \mathrm{~d} t .
\end{aligned}
$$

Now, we will be concerned with the integral of $\mathrm{e}^{-2 s \alpha^{*}+2 s \hat{\alpha}} \hat{\xi}^{-11 / 2}\left|u_{2, t}\right|^{2}$. This estimate is the hardest and most important part of the present paper.

First, we integrate by parts twice with respect to $t$ and we obtain

$$
\begin{aligned}
& s^{-11 / 2} \lambda^{2} \iint_{\omega_{2} \times(0, T)} \mathrm{e}^{-2 s \alpha^{*}+2 s \hat{\alpha}} \hat{\xi}^{-11 / 2}\left|u_{2, t}\right|^{2} \mathrm{~d} x \mathrm{~d} t=\frac{1}{2} s^{-11 / 2} \lambda^{2} \iint_{\omega_{2} \times(0, T)}\left(\mathrm{e}^{-2 s \alpha^{*}+2 s \hat{\alpha}} \hat{\xi}^{-11 / 2}\right) t t\left|u_{2}\right|^{2} \mathrm{~d} x \mathrm{~d} t \\
& -s^{-11 / 2} \lambda^{2} \iint_{\omega_{2} \times(0, T)} \mathrm{e}^{-2 s \alpha^{*}+2 s \hat{\alpha}} \hat{\xi}^{-11 / 2} u_{2, t t} \cdot u_{2} \mathrm{~d} x \mathrm{~d} t .
\end{aligned}
$$

It is not difficult to see that the weight function $\left(s^{-11 / 2} \lambda^{2} \mathrm{e}^{-2 s \alpha^{*}+2 s \hat{\alpha}} \hat{\xi}^{-11 / 2}\right)_{t t}$ is bounded for a suitable choice of $s$ and $\lambda$, so we concentrate in estimating the last integral in (37). Let us hence introduce the function

$$
\eta^{*}=s^{-11 / 2} \lambda^{-5} \mathrm{e}^{-2 s \alpha^{*}+2 s \hat{\alpha}} \hat{\xi}^{-11 / 2} .
$$

Using Hölder's inequality, we deduce that

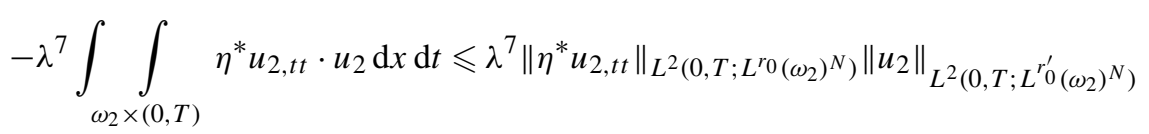

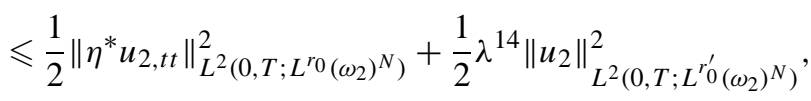

where $1<r_{0}<r$ if $N=2$ and $6 / 5<r_{0}<r$ if $N=3$ ( $r$ was defined in (6)).

Let us first deal with the last term in the right-hand side of (38). We introduce an open set $\omega_{3} \ni \omega_{2}$ and a cut-off function $\zeta \in C^{2}\left(\omega_{3}\right)$ such that

$$
\operatorname{supp} \zeta \subset \omega_{3} \text { and } \zeta \equiv 1 \text { in } \omega_{2} .
$$

Then,

$$
\left\|u_{2}\right\|_{L^{2}\left(0, T ; L^{\left.r_{0}^{\prime}\left(\omega_{2}\right)^{N}\right)}\right.}^{2} \leqslant C\left\|\Delta\left(\zeta u_{2}\right)\right\|_{L^{2}\left(0, T ; L^{2}\left(\omega_{3}\right)^{N}\right)}^{2}=C\left\|u_{2} \Delta \zeta+2 \nabla \zeta \cdot \nabla u_{2}+\zeta \Delta u_{2}\right\|_{L^{2}\left(0, T ; L^{2}\left(\omega_{3}\right)^{N}\right)}^{2},
$$

since $\left(H^{2}\left(\omega_{3}\right) \cap H_{0}^{1}\left(\omega_{3}\right)\right)^{N}$ is continuously imbedded in $L^{r_{0}^{\prime}}\left(\omega_{3}\right)^{N}$, for $r_{0}^{\prime}<\infty$.

Now, we can use the local estimate contained in the Appendix (at the end of this paper) in order to get a bound of $\left\|\zeta \Delta u_{2}\right\|_{L^{2}\left(0, T ; L^{2}\left(\omega_{3}\right)^{N}\right)}^{2}$. In fact, since $u_{2}(T)=0$, it suffices to apply inequality (87) with $\varphi=u_{2}, \hat{\eta}=1, g_{1}=-\eta^{\prime} \varphi$, $\omega_{2}=\omega_{3}$ and $\omega_{4}=\omega_{5}$, where $\omega_{4}$ and $\omega_{5}$ are open sets verifying

$$
\omega_{3} \Subset \omega_{4} \Subset \omega_{5} \Subset \omega .
$$

This gives 


$$
\left\|u_{2}\right\|_{L^{2}\left(0, T ; L^{\left.r_{0}^{\prime}\left(\omega_{2}\right)^{N}\right)}\right.}^{2} \leqslant C\left(1+\|\bar{y}\|_{\infty}^{2}+\|\bar{\theta}\|_{\infty}^{2}\right)(1+T) \iint_{\omega_{5} \times(0, T)}\left(\left|u_{2}\right|^{2}+\left|\nabla u_{2}\right|^{2}+\left|\nabla w_{2}\right|^{2}+\left|\eta^{\prime} \varphi\right|^{2}\right) \mathrm{d} x \mathrm{~d} t .
$$

From the definitions of $u_{2}$ and $w_{2}$, we also find that

$$
\left|u_{2}\right|^{2}+\left|\nabla u_{2}\right|^{2} \leqslant 2\left(\left|u_{1}\right|^{2}+\left|\nabla u_{1}\right|^{2}+|\eta|^{2}\left(|\varphi|^{2}+|\nabla \varphi|^{2}\right)\right)
$$

and

$$
\left|\nabla w_{2}\right|^{2} \leqslant 2\left(\left|\nabla w_{1}\right|^{2}+|\eta|^{2}|\nabla \psi|^{2}\right) .
$$

Consequently, viewing $\left(u_{1}, w_{1}\right)$ as the weak solution of (33) and using again global estimates, we see that

$$
\begin{aligned}
& \left\|u_{2}\right\|_{L^{2}\left(0, T ; L^{\left.r_{0}^{\prime}\left(\omega_{2}\right)^{N}\right)}\right.}^{2} \leqslant C\left(1+\|\bar{y}\|_{\infty}^{2}+\|\bar{\theta}\|_{\infty}^{2}\right)(1+T) \mathrm{e}^{C T\left(1+\|\bar{y}\|_{\infty}^{2}+\|\bar{\theta}\|_{\infty}^{2}\right)} \\
& \times\left(\left\|\eta g_{1}\right\|_{L^{2}(Q)^{N}}^{2}+\left\|\eta g_{2}\right\|_{L^{2}(Q)}^{2}+\iint_{\omega_{5} \times(0, T)}\left(|\eta|^{2}+\left|\eta^{\prime}\right|^{2}\right)|\varphi|^{2} \mathrm{~d} x \mathrm{~d} t\right. \\
& \left.+\iint_{\omega_{5} \times(0, T)}|\eta|^{2}\left(|\nabla \varphi|^{2}+|\nabla \psi|^{2}\right) \mathrm{d} x \mathrm{~d} t\right) .
\end{aligned}
$$

Let us now estimate the norm involving $u_{2, t t}$ in (38). The triple $(u, q, w):=\left(\eta^{*} u_{2, t}, \eta^{*} q_{2, t}, \eta^{*} w_{2, t}\right)$ satisfies

$$
\begin{cases}-u_{t}-\Delta u-D u \bar{y}+\nabla q=G_{1}+\bar{\theta} \nabla w & \text { in } Q, \\ -w_{t}-\Delta w-\bar{y} \cdot \nabla w=G_{2} & \text { in } Q, \\ \nabla \cdot u=0 & \text { in } Q, \\ u=0, \quad w=0 & \text { on } \Sigma, \\ u(T)=0, \quad w(T)=0 & \text { in } \Omega,\end{cases}
$$

where

$$
G_{1}=-\eta^{*} \eta^{\prime \prime} \varphi-\eta^{*} \eta^{\prime} \varphi_{t}+\eta^{*} D u_{2} \bar{y}_{t}+\eta^{*} \bar{\theta}_{t} \nabla w_{2}-\left(\eta^{*}\right)^{\prime} u_{2, t}
$$

and

$$
G_{2}=-\eta^{*} \eta^{\prime \prime} \psi-\eta^{*} \eta^{\prime} \psi_{t}+\eta^{*} \eta \varphi_{N, t}+\eta^{*} \eta^{\prime} \varphi_{N}+\eta^{*} \bar{y}_{t} \cdot \nabla w_{2}-\left(\eta^{*}\right)^{\prime} w_{2, t} .
$$

The fact that $(u, q, w)$ fulfills $(40)$ is not direct. For instance, this can be verified by first considering a sequence $\left\{\bar{y}^{n}, \bar{\theta}^{n}\right\}$ of regular functions satisfying

$$
\left(\bar{y}^{n}, \bar{\theta}^{n}\right) \rightarrow(\bar{y}, \bar{\theta}) \quad \text { weakly star in } L^{\infty}(Q)^{N}
$$

and

$$
\left(\bar{y}_{t}^{n}, \bar{\theta}_{t}^{n}\right) \rightarrow\left(\bar{y}_{t}, \bar{\theta}_{t}\right) \quad \text { weakly in } L^{2}\left(0, T ; L^{r}(\Omega)^{N}\right) .
$$

Then, one can easily prove the existence and uniqueness of a solution $\left(u^{n}, q^{n}, w^{n}\right)$ to (40) with $(\bar{y}, \bar{\theta})$ replaced by $\left(\bar{y}^{n}, \bar{\theta}^{n}\right)$. Finally, we pass to the limit and deduce that $(u, q, w)$ is actually the solution of (40).

In order to obtain an estimate of $u_{t}$ in $L^{2}\left(0, T ; L^{r_{0}}(\Omega)^{N}\right)$, we first give the estimates of $u$ and $w$ as weak solutions. We have

$$
\begin{aligned}
& \|u\|_{L^{2}(0, T ; V)}+\|w\|_{L^{2}\left(0, T ; H^{1}(\Omega)\right)} \\
& \quad \leqslant C \mathrm{e}^{C T\left(1+\|\bar{y}\|_{\infty}^{2}+\|\bar{\theta}\|_{\infty}^{2}\right)}\left(\left\|G_{1}\right\|_{L^{2}\left(0, T ; H^{-1}(\Omega)^{N}\right)}+\left\|G_{2}\right\|_{L^{2}\left(0, T ; H^{-1}(\Omega)\right)}\right) .
\end{aligned}
$$

For the moment, let us assume that $\left(\eta^{*} D u_{2} \bar{y}_{t}\right)_{i}, \eta^{*} \bar{y}_{t} \cdot \nabla w_{2},\left(\eta^{*} \bar{\theta}_{t} \nabla w_{2}\right)_{i} \in L^{2}\left(0, T ; L^{r_{0}}(\Omega)\right)$; this will be proved in the next paragraph.

At this point, we recall a result proved in [7] about the regularity of solutions of Stokes systems: 
Lemma 1. Let $1<q_{1}, q_{2}<\infty$ and suppose that $u^{0} \in W^{1, q_{2}}(\Omega)^{N}$ and $f \in L^{q_{1}}\left(0, T ; L^{q_{2}}(\Omega)^{N}\right)$. Then, the weak solution $u \in L^{2}(0, T ; V) \cap L^{\infty}(0, T ; H)$ of system

$$
\begin{cases}u_{t}-\Delta u+\nabla \pi=f & \text { in } Q, \\ \nabla \cdot u=0 & \text { in } Q, \\ u=0 & \text { on } \Sigma, \\ u(0)=u^{0} & \text { in } \Omega\end{cases}
$$

actually verifies, together with a pressure $\pi$, that

$$
(u, \nabla \pi) \in\left(L^{q_{1}}\left(0, T ; W^{2, q_{2}}(\Omega)^{N}\right) \cap W^{1, q_{1}}\left(0, T ; L^{q_{2}}(\Omega)^{N}\right)\right) \times L^{q_{1}}\left(0, T ; L^{q_{2}}(\Omega)^{N}\right) .
$$

Moreover, there exists a positive constant $C$ just depending on $\Omega$ such that

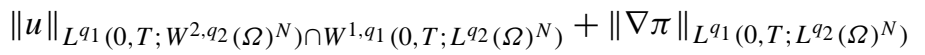

$$
\begin{aligned}
& \leqslant C\left(\|f\|_{L^{q_{1}\left(0, T ; L^{q_{2}}(\Omega)^{N}\right)}}+\left\|u^{0}\right\|_{W^{1, q_{2}(\Omega)^{N}}}\right) .
\end{aligned}
$$

Remark 3. In fact, the exact result proved in [7] just considers solenoidal right-hand sides $f$. However, from this result and the application of Helmotz's decomposition in the spaces $L^{q_{1}}\left(0, T ; L^{q_{2}}(\Omega)^{N}\right)$, Lemma 1 readily follows.

Under these conditions, we can apply Lemma 1 and deduce, among other properties, that $u_{t} \in L^{2}\left(0, T ; L^{r_{0}}(\Omega)^{N}\right)$ and

$$
\left\|u_{t}\right\|_{L^{2}\left(0, T ; L^{\left.r_{0}(\Omega)^{N}\right)}\right.} \leqslant C\left\|G_{1}+\bar{\theta} \nabla w+D u \bar{y}\right\|_{L^{2}\left(0, T ; L^{\left.r_{0}(\Omega)^{N}\right)}\right.}
$$

for a positive constant $C$ depending on $\Omega$ but not on $T$. Since $L^{r_{0}}(\Omega)$ is continuously imbedded in $H^{-1}(\Omega),(41)$ and (42) yield

$$
\begin{aligned}
& \left\|\eta^{*} u_{2, t t}\right\|_{L^{2}\left(0, T ; L^{\left.r_{0}(\Omega)^{N}\right)}\right.} \leqslant C\left(1+\|\bar{y}\|_{\infty}+\|\bar{\theta}\|_{\infty}\right) \mathrm{e}^{C T\left(1+\|\bar{y}\|_{\infty}^{2}+\|\bar{\theta}\|_{\infty}^{2}\right)}\left(\left\|\eta^{*} \eta^{\prime \prime} \varphi\right\|_{L^{2}(Q)^{N}}+\left\|\eta^{*} \eta^{\prime} \varphi_{t}\right\|_{L^{2}(Q)^{N}}\right. \\
& +\left\|\left(\eta^{*}\right)^{\prime} u_{2, t}\right\|_{L^{2}(Q)^{N}}+\left\|\eta^{*} D u_{2} \bar{y}_{t}\right\|_{L^{2}\left(0, T ; L^{\left.r_{0}(\Omega)^{N}\right)}\right.}+\left\|\eta^{*} \bar{\theta}_{t} \nabla w_{2}\right\|_{L^{2}\left(0, T ; L^{\left.r_{0}(\Omega)^{N}\right)}\right.} \\
& +\left\|\eta^{*} \eta^{\prime \prime} \psi\right\|_{L^{2}(Q)}+\left\|\eta^{*} \eta^{\prime} \psi_{t}\right\|_{L^{2}(Q)}+\left\|\eta^{*} \eta \varphi_{N, t}\right\|_{L^{2}(Q)}+\left\|\eta^{*} \eta^{\prime} \varphi_{N}\right\|_{L^{2}(Q)} \\
& \left.+\left\|\eta^{*} \bar{y}_{t} \cdot \nabla w_{2}\right\|_{L^{2}\left(0, T ; L^{\left.r_{0}(\Omega)\right)}\right.}+\left\|\left(\eta^{*}\right)^{\prime} w_{2, t}\right\|_{L^{2}(Q)}\right) .
\end{aligned}
$$

From (37)-(39) and this last inequality, we have

$$
\begin{aligned}
s^{-11 / 2} \lambda^{2} \int & \int_{\omega_{2} \times(0, T)} \mathrm{e}^{-2 s \alpha^{*}+2 s \hat{\alpha}} \hat{\xi}^{-11 / 2}\left|u_{2, t}\right|^{2} \mathrm{~d} x \mathrm{~d} t \\
\leqslant & C\left(1+\|\bar{y}\|_{\infty}^{2}+\|\bar{\theta}\|_{\infty}^{2}\right) \mathrm{e}^{C T\left(1+\|\bar{y}\|_{\infty}^{2}+\|\bar{\theta}\|_{\infty}^{2}\right)}\left(\lambda ^ { 1 4 } ( 1 + T ) \left(\left\|\eta g_{1}\right\|_{L^{2}(Q)^{N}}^{2}+\left\|\eta g_{2}\right\|_{L^{2}(Q)}^{2}\right.\right. \\
& \left.+\|\eta \varphi\|_{L^{2}\left(0, T ; L^{2}\left(\omega_{5}\right)^{N}\right)}^{2}+\left\|\eta^{\prime} \varphi\right\|_{L^{2}\left(0, T ; L^{2}\left(\omega_{5}\right)^{N}\right)}^{2}+\|\eta \nabla \varphi\|_{L^{2}\left(0, T ; L^{2}\left(\omega_{5}\right)^{N}\right)}^{2}+\|\eta \nabla \psi\|_{L^{2}\left(0, T ; L^{2}\left(\omega_{5}\right)^{N}\right)}^{2}\right) \\
& +\left\|\eta^{*} \eta^{\prime \prime} \varphi\right\|_{L^{2}(Q)^{N}}^{2}+\left\|\eta^{*} \eta^{\prime} \varphi_{t}\right\|_{L^{2}(Q)^{N}}^{2}+\left\|\left(\eta^{*}\right)^{\prime} u_{2, t}\right\|_{L^{2}(Q)^{N}}^{2}+\left\|\eta^{*} D u_{2} \bar{y}_{t}\right\|_{L^{2}\left(0, T ; L^{\prime}(\Omega)^{N}\right)}^{2} \\
& +\left\|\eta^{*} \bar{\theta}_{t} \nabla w_{2}\right\|_{L^{2}\left(0, T ; L^{r}(\Omega)^{N}\right)}^{2}+\left\|\eta^{*} \eta^{\prime \prime} \psi\right\|_{L^{2}(Q)}^{2}+\left\|\eta^{*} \eta^{\prime} \psi_{t}\right\|_{L^{2}(Q)}^{2}+\left\|\eta^{*} \eta \varphi_{N, t}\right\|_{L^{2}(Q)}^{2} \\
& \left.+\left\|\eta^{*} \eta^{\prime} \varphi_{N}\right\|_{L^{2}(Q)}^{2}+\left\|\eta^{*} \bar{y}_{t} \cdot \nabla w_{2}\right\|_{L^{2}\left(0, T ; L^{r}(\Omega)\right)}^{2}+\left\|\left(\eta^{*}\right)^{\prime} w_{2, t}\right\|_{L^{2}(Q)}^{2}\right) .
\end{aligned}
$$

To finish this paragraph, we combine (35), (36) and (43) and we obtain the following estimate of $\varphi_{t}$ : 


$$
\begin{aligned}
s^{2} \lambda^{2} \int_{\omega_{2} \times(0, T)} \mathrm{e}^{-2 s \hat{\alpha}} \hat{\xi}^{2}\left|\varphi_{t}\right|^{2} \mathrm{~d} x \mathrm{~d} t \leqslant & C\left(1+\|\bar{y}\|_{\infty}^{2}+\|\bar{\theta}\|_{\infty}^{2}\right) \mathrm{e}^{C T\left(1+\|\bar{y}\|_{\infty}^{2}+\|\bar{\theta}\|_{\infty}^{2}\right)}\left(\lambda ^ { 1 4 } ( 1 + T ) \left(\left\|\eta g_{1}\right\|_{L^{2}(Q)^{N}}^{2}\right.\right. \\
& +\left\|\eta g_{2}\right\|_{L^{2}(Q)}^{2}+\|\eta \varphi\|_{L^{2}\left(0, T ; L^{2}\left(\omega_{5}\right)^{N}\right)}^{2}+\left\|\eta^{\prime} \varphi\right\|_{L^{2}\left(0, T ; L^{2}\left(\omega_{5}\right)^{N}\right)}^{2} \\
& \left.+\|\eta \nabla \varphi\|_{L^{2}\left(0, T ; L^{2}\left(\omega_{5}\right)^{N}\right)}^{2}+\|\eta \nabla \psi\|_{L^{2}\left(0, T ; L^{2}\left(\omega_{5}\right)^{N}\right)}^{2}\right) \\
& +\left\|\eta^{*} \eta^{\prime \prime} \varphi\right\|_{L^{2}(Q)^{N}}^{2}+\left\|\eta^{*} \eta^{\prime} \varphi_{t}\right\|_{L^{2}(Q)^{N}}^{2}+\left\|\left(\eta^{*}\right)^{\prime} u_{2, t}\right\|_{L^{2}(Q)^{N}}^{2} \\
& +\left\|\eta^{*} D u_{2} \bar{y}_{t}\right\|_{L^{2}\left(0, T ; L^{r} 0(\Omega)^{N}\right)}^{2}+\left\|\eta^{*} \bar{\theta}_{t} \nabla w_{2}\right\|_{L^{2}\left(0, T ; L^{2}(\Omega)^{N}\right)}^{2} \\
& +\left\|\eta^{*} \eta^{\prime \prime} \psi\right\|_{L^{2}(Q)}^{2}+\left\|\eta^{*} \eta^{\prime} \psi_{t}\right\|_{L^{2}(Q)}^{2}+\left\|\eta^{*} \eta \varphi_{N, t}\right\|_{L^{2}(Q)}^{2} \\
& +\left\|\eta^{*} \eta^{\prime} \varphi_{N}\right\|_{L^{2}(Q)}^{2}+\left\|\eta^{*} \bar{y}_{t} \cdot \nabla w_{2}\right\|_{L^{2}\left(0, T ; L^{\prime}(\Omega)\right)}^{2} \\
& \left.+\left\|\left(\eta^{*}\right)^{\prime} w_{2, t}\right\|_{L^{2}(Q)}^{2}\right) .
\end{aligned}
$$

2.2.2. Estimate of $\left(\eta^{*} D u_{2} \bar{y}_{t}\right)_{i}, \eta^{*} \bar{y}_{t} \cdot \nabla w_{2}$ and $\left(\eta^{*} \bar{\theta}_{t} \nabla w_{2}\right)_{i}$ in $L^{2}\left(0, T ; L^{r_{0}}(\Omega)\right)$

The strategy we follow in this step is to deduce an estimate of $\left(\eta^{*} u_{2}\right)_{i}$ and $\eta^{*} w_{2}$ in $L^{\infty}\left(0, T ; W^{1, l}(\Omega)\right)$ for every $l<\infty$.

Observe that, once this is achieved, from the fact that $\bar{y}$ and $\bar{\theta}$ satisfy (5) and the choice we have made of $r_{0}$, it is easy to obtain an estimate of $\left(\eta^{*} D u_{2} \bar{y}_{t}\right)_{i}, \eta^{*} \bar{y}_{t} \cdot \nabla w_{2}$ and $\left(\eta^{*} \bar{\theta}_{t} \nabla w_{2}\right)_{i}$ in $L^{2}\left(0, T ; L^{r_{0}}(\Omega)\right)$.

The functions $\left(\eta^{*} u_{2}, \eta^{*} q_{2}, \eta^{*} w_{2}\right)$ solve the following system:

$$
\begin{cases}-\left(\eta^{*} u_{2}\right)_{t}-\Delta\left(\eta^{*} u_{2}\right)-D\left(\eta^{*} u_{2}\right) \bar{y}+\nabla\left(\eta^{*} q_{2}\right)=-\left(\eta^{*}\right)^{\prime} u_{2}-\eta^{*} \eta^{\prime} \varphi+\bar{\theta} \nabla\left(\eta^{*} w_{2}\right) & \text { in } Q, \\ -\left(\eta^{*} w_{2}\right)_{t}-\Delta\left(\eta^{*} w_{2}\right)-\bar{y} \cdot \nabla\left(\eta^{*} w_{2}\right)=-\left(\eta^{*}\right)^{\prime} w_{2}-\eta^{*} \eta^{\prime} \psi+\eta^{*} \eta \varphi_{N} & \text { in } Q, \\ \nabla \cdot\left(\eta^{*} u_{2}\right)=0 & \text { in } Q, \\ \eta^{*} u_{2}=0, \quad \eta^{*} w_{2}=0 & \text { on } \Sigma, \\ \left(\eta^{*} u_{2}\right)(T)=0, \quad\left(\eta^{*} w_{2}\right)(T)=0 & \text { in } \Omega .\end{cases}
$$

From well known interpolation inequalities we deduce that, for $N \leqslant 3$,

$$
L^{2}\left(0, T ; H^{2}(\Omega)^{N}\right) \cap L^{\infty}\left(0, T ; H^{1}(\Omega)^{N}\right) \subset L^{k_{1}}\left(0, T ; L^{k_{2}}(\Omega)^{N}\right)
$$

with

$$
\frac{2}{k_{1}}+\frac{6}{k_{2}}=1
$$

and

$$
L^{2}\left(0, T ; L^{6}(\Omega)^{N}\right) \cap L^{\infty}\left(0, T ; L^{2}(\Omega)^{N}\right) \subset L^{k_{3}}\left(0, T ; L^{k_{4}}(\Omega)^{N}\right)
$$

with

$$
\frac{4 / 3}{k_{3}}+\frac{2}{k_{4}}=1
$$

We are going to deduce the $L^{\infty}\left(0, T ; W^{1, l}(\Omega)\right)$ regularity for $\left(\eta^{*} u_{2}, \eta^{*} w_{2}\right)$ after a bootstrap argument with two steps.

- First, watching $\left(\eta^{*} u_{2}, \eta^{*} w_{2}\right)$ as the strong solution of (45), we have

$$
\left(D\left(\eta^{*} u_{2}\right) \bar{y}\right)_{i}, \bar{\theta} \nabla\left(\eta^{*} w_{2}\right) \in L^{2}\left(0, T ; L^{6}(\Omega)\right) \cap L^{\infty}\left(0, T ; L^{2}(\Omega)\right),
$$

so they belong to the space $L^{k_{3}}\left(0, T ; L^{k_{4}}(\Omega)\right)$.

Using again Lemma 1 , we deduce that $\eta^{*} u_{2} \in L^{k_{3}}\left(0, T ; W^{2, k_{4}}(\Omega)^{N}\right), \eta^{*} w_{2} \in L^{k_{3}}\left(0, T ; W^{2, k_{4}}(\Omega)\right)$ and 


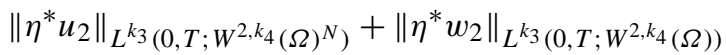

$$
\begin{aligned}
& \leqslant C\left(\left\|-\left(\eta^{*}\right)^{\prime} u_{2}-\eta^{*} \eta^{\prime} \varphi+\eta^{*} D u_{2} \bar{y}+\eta^{*} \bar{\theta} \nabla w_{2}\right\|_{L^{k_{3}\left(0, T ; L^{k_{4}}(\Omega)^{N}\right)}}\right. \\
& \left.+\left\|-\left(\eta^{*}\right)^{\prime} w_{2}-\eta^{*} \eta^{\prime} \psi+\eta^{*} \eta \varphi_{N}+\eta^{*} \bar{y} \cdot \nabla w_{2}\right\|_{\left.L^{k_{3}\left(0, T ; L^{\left.k_{4}(\Omega)\right)}\right.}\right)}\right) .
\end{aligned}
$$

Accordingly, $\left(\eta^{*} D u_{2}\right)_{i}, \eta^{*} \nabla w_{2} \in L^{k_{3}}\left(0, T ; W^{1, k_{4}}(\Omega)^{N}\right)$. In this argument, $k_{4}$ can be any number satisfying $3 \leqslant$ $k_{4}<6$. This gives $\left(\eta^{*} D u_{2}\right)_{i}, \eta^{*} \nabla w_{2} \in L^{k_{1}}\left(0, T ; L^{k_{2}}(\Omega)^{N}\right)$, with $k_{1}=k_{3}$ given by (47) and $k_{2}=l>6$.

- Secondly, we use that $\eta^{*} D u_{2} \bar{y}, \eta^{*} \bar{\theta} \nabla w_{2} \in L^{k_{3}}\left(0, T ; L^{l}(\Omega)^{N}\right)$ and we obtain from Lemma 1 (among other things) that $\nabla\left(\eta^{*} q_{2}\right) \in L^{k_{3}}\left(0, T ; L^{l}(\Omega)^{N}\right)$ and

$$
\left\|\nabla\left(\eta^{*} q_{2}\right)\right\|_{L^{k_{3}\left(0, T ; L^{l}(\Omega)^{N}\right)}} \leqslant C\left\|-\left(\eta^{*}\right)^{\prime} u_{2}-\eta^{*} \eta^{\prime} \varphi+\eta^{*} D u_{2} \bar{y}+\eta^{*} \bar{\theta} \nabla w_{2}\right\|_{L^{k_{3}\left(0, T ; L^{l}(\Omega)^{N}\right)}} .
$$

Then, we take into account (48) and we get

$$
\begin{aligned}
& \left\|\nabla\left(\eta^{*} q_{2}\right)\right\|_{L^{k_{3}\left(0, T ; L^{l}(\Omega)^{N}\right)}} \leqslant C\left(1+\|\bar{y}\|_{\infty}^{2}+\|\bar{\theta}\|_{\infty}^{2}\right)\left(\left\|\left(\eta^{*}\right)^{\prime} \Delta u_{2}\right\|_{L^{2}(Q)^{N}}+\left\|\left(\left(\eta^{*}\right)^{\prime} u_{2}\right)_{t}\right\|_{L^{2}(Q)^{N}}\right. \\
& +\left\|\eta^{*} \Delta u_{2}\right\|_{L^{2}(Q)^{N}}+\left\|\left(\eta^{*} u_{2}\right)_{t}\right\|_{L^{2}(Q)^{N}} \\
& +\left\|\eta^{*} \eta^{\prime} \Delta \varphi\right\|_{L^{2}(Q)^{N}}+\left\|\left(\eta^{*} \eta^{\prime} \varphi\right)_{t}\right\|_{L^{2}(Q)^{N}}+\left\|\left(\eta^{*}\right)^{\prime} w_{2}\right\|_{L^{2}(Q)} \\
& +\left\|\left(\eta^{*}\right)^{\prime} \nabla w_{2}\right\|_{L^{2}(Q)^{N}}+\left\|\eta^{*} \Delta w_{2}\right\|_{L^{2}(Q)}+\left\|\left(\eta^{*} w_{2}\right)_{t}\right\|_{L^{2}(Q)} \\
& +\left\|\eta^{*} \eta^{\prime} \psi\right\|_{L^{2}(Q)}+\left\|\eta^{*} \eta^{\prime} \nabla \psi\right\|_{L^{2}(Q)^{N}}+\left\|\eta^{*} \eta \varphi_{N}\right\|_{L^{2}(Q)} \\
& +\left\|\eta^{*} \eta \nabla \varphi_{N}\right\|_{\left.L^{2}(Q)^{N}\right)} \text {. }
\end{aligned}
$$

Let us see that this suffices to ensure that $\left(\eta^{*} u_{2}\right)_{i}, \eta^{*} w_{2} \in L^{\infty}\left(0, T ; W^{1, l}(\Omega)\right)$. To this end, we can restrict ourselves to the general case of a heat system with zero Dirichlet boundary conditions, null initial condition and a right-hand side in $L^{k_{3}}\left(0, T ; L^{l}(\Omega)\right)$ with $k_{3}>2$.

For instance, for the case of $\eta^{*} u_{2}$ we have (see [10] for more details)

$$
\left\|\eta^{*} u_{2}(t)\right\|_{W^{1, l}(\Omega)^{N}} \leqslant C \int_{0}^{t}(t-s)^{-1 / 2}\|B(s)\|_{L^{l}(\Omega)^{N}} \mathrm{~d} s \quad \forall t \in(0, T)
$$

where $B$ denotes the right-hand side. Since $\|B(\cdot)\|_{L^{l}(\Omega)^{N}} \in L^{k_{3}}(0, T)$ and $k_{3}>2$, Young's inequality tells us that $\left\|\eta^{*} u_{2}(\cdot)\right\|_{W^{1, l}(\Omega)^{N}} \in L^{\infty}(0, T)$ and

$$
\left\|\eta^{*} u_{2}\right\|_{L^{\infty}\left(0, T ; W^{1, l}(\Omega)^{N}\right)} \leqslant C\left(1-k_{3}^{\prime} / 2\right)^{-1 / k_{3}^{\prime}} T^{-1 / 2+1 / k_{3}^{\prime}}\|B\|_{L^{k_{3}}\left(0, T ; L^{l}(\Omega)^{N}\right)} .
$$

The same is also true for $\eta^{*} w_{2}$. Then, from (49), we obtain the desired regularity for $\eta^{*} u_{2}$ and $\eta^{*} w_{2}$ and

$$
\begin{aligned}
&\left\|\eta^{*} u_{2}\right\|_{L^{\infty}\left(0, T ; W^{1, l}(\Omega)^{N}\right)}+\left\|\eta^{*} w_{2}\right\|_{L^{\infty}\left(0, T ; W^{1, l}(\Omega)\right)} \\
& \leqslant C T^{-1 / 2+1 / k_{3}^{\prime}}\left(1+\|\bar{y}\|_{\infty}^{2}+\|\bar{\theta}\|_{\infty}^{2}\right)\left(\left\|\left(\eta^{*}\right)^{\prime} \Delta u_{2}\right\|_{L^{2}(Q)^{N}}+\left\|\left(\left(\eta^{*}\right)^{\prime} u_{2}\right)_{t}\right\|_{L^{2}(Q)^{N}}+\left\|\eta^{*} \Delta u_{2}\right\|_{L^{2}(Q)^{N}}\right. \\
&+\left\|\left(\eta^{*} u_{2}\right)_{t}\right\|_{L^{2}(Q)^{N}}+\left\|\eta^{*} \eta^{\prime} \Delta \varphi\right\|_{L^{2}(Q)^{N}}+\left\|\left(\eta^{*} \eta^{\prime} \varphi\right)_{t}\right\|_{L^{2}(Q)^{N}}+\left\|\left(\eta^{*}\right)^{\prime} \Delta w_{2}\right\|_{L^{2}(Q)} \\
&+\left\|\left(\left(\eta^{*}\right)^{\prime} w_{2}\right)_{t}\right\|_{L^{2}(Q)^{N}}+\left\|\eta^{*} \Delta w_{2}\right\|_{L^{2}(Q)}+\left\|\left(\eta^{*} w_{2}\right)_{t}\right\|_{L^{2}(Q)}+\left\|\eta^{*} \eta^{\prime} \Delta \psi\right\|_{L^{2}(Q)}+\left\|\left(\eta^{*} \eta^{\prime} \psi\right)_{t}\right\|_{L^{2}(Q)} \\
&\left.+\left\|\eta^{*} \eta \Delta \varphi_{N}\right\|_{L^{2}(Q)}+\left\|\left(\eta^{*} \eta \varphi_{N}\right)_{t}\right\|_{L^{2}(Q)}\right) .
\end{aligned}
$$

As mentioned above, combining (50) and the regularity of $\bar{y}$ and $\bar{\theta}$ (given in (5)) we find that $\left(\eta^{*} D u_{2} \bar{y}_{t}\right)_{i}$, $\eta^{*} \bar{y}_{t} \cdot \nabla w_{2},\left(\eta^{*} \bar{\theta}_{t} \nabla w_{2}\right)_{i} \in L^{2}\left(0, T ; L^{r_{0}}(\Omega)\right)$ and 


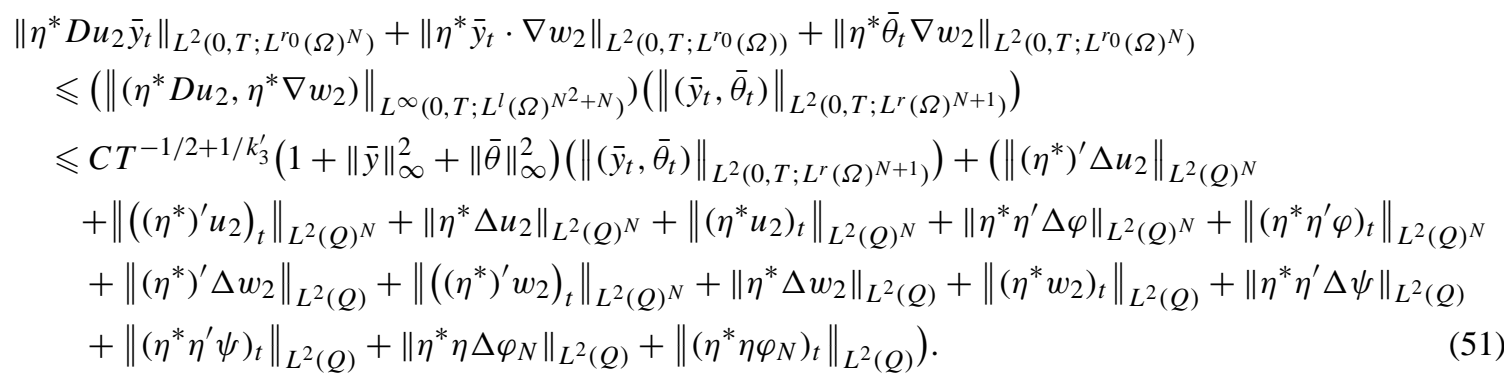

Then, we put together inequalities (44) and (51) and we use that

$$
-1+\frac{2}{k_{3}^{\prime}} \leqslant \frac{1}{2}
$$

We obtain:

$$
\begin{aligned}
& s^{2} \lambda^{2} \int_{\omega_{2} \times(0, T)} \mathrm{e}^{-2 s \hat{\alpha}} \hat{\xi}^{2}\left|\varphi_{t}\right|^{2} \mathrm{~d} x \mathrm{~d} t \leqslant C\left(1+\|\bar{y}\|_{\infty}^{6}+\|\bar{\theta}\|_{\infty}^{6}\right)\left\|\left(\bar{y}_{t}, \bar{\theta}_{t}\right)\right\|_{L^{2}\left(0, T ; L^{r}(\Omega)^{N+1}\right)}^{2} \mathrm{e}^{C T\left(1+\|\bar{y}\|_{\infty}^{2}+\|\bar{\theta}\|_{\infty}^{2}\right)} \\
& \quad \times\left(\lambda ^ { 1 4 } ( 1 + T ) \left(\left\|\eta g_{1}\right\|_{L^{2}(Q)^{N}}^{2}+\left\|\eta g_{2}\right\|_{L^{2}(Q)}^{2}+\|\eta \varphi\|_{L^{2}\left(0, T ; L^{2}\left(\omega_{5}\right)^{N}\right)}^{2}\right.\right. \\
& \quad+\|\eta \nabla \varphi\|_{L^{2}\left(0, T ; L^{2}\left(\omega_{5}\right)^{N}\right)}^{2}+\left\|\eta^{\prime} \varphi\right\|_{L^{2}\left(0, T ; L^{2}\left(\omega_{5}\right)^{N}\right)}^{2} \\
& \left.\left.\quad+\|\eta \nabla \psi\|_{L^{2}\left(0, T ; L^{2}\left(\omega_{5}\right)^{N}\right)}^{2}\right)+\left(1+T^{1 / 2}\right)\left(J_{1}(\varphi)+J_{2}(\psi)+J_{3}\left(u_{2}\right)+J_{4}\left(w_{2}\right)\right)\right)
\end{aligned}
$$

where

$$
\begin{aligned}
J_{1}(\varphi)= & \left\|\eta^{*} \eta^{\prime \prime} \varphi\right\|_{L^{2}(Q)^{N}}^{2}+\left\|\left(\eta^{*}\right)^{\prime} \eta^{\prime} \varphi\right\|_{L^{2}(Q)^{N}}^{2}+\left\|\eta^{*} \eta^{\prime} \varphi_{t}\right\|_{L^{2}(Q)^{N}}^{2}+\left\|\eta^{*} \eta^{\prime} \Delta \varphi\right\|_{L^{2}(Q)^{N}}^{2}+\left\|\eta^{*} \eta \varphi_{N, t}\right\|_{L^{2}(Q)}^{2} \\
& +\left\|\eta^{*} \eta^{\prime} \varphi_{N}\right\|_{L^{2}(Q)}^{2}+\left\|\left(\eta^{*}\right)^{\prime} \eta \varphi_{N}\right\|_{L^{2}(Q)}+\left\|\eta^{*} \eta \Delta \varphi_{N}\right\|_{L^{2}(Q)}, \\
J_{2}(\psi)= & \left\|\eta^{*} \eta^{\prime \prime} \psi\right\|_{L^{2}(Q)}^{2}+\left\|\eta^{*} \eta^{\prime} \psi_{t}\right\|_{L^{2}(Q)}^{2}+\left\|\eta^{*} \eta^{\prime} \Delta \psi\right\|_{L^{2}(Q)^{N}}+\left\|\left(\eta^{*}\right)^{\prime} \eta^{\prime} \psi\right\|_{L^{2}(Q)}, \\
J_{3}\left(u_{2}\right)= & \left\|\eta^{*} \Delta u_{2}\right\|_{L^{2}(Q)^{N}}^{2}+\left\|\left(\eta^{*}\right)^{\prime} \Delta u_{2}\right\|_{L^{2}(Q)^{N}}^{2}+\left\|\left(\eta^{*}\right)^{\prime} u_{2, t}\right\|_{L^{2}(Q)^{N}}^{2}+\left\|\left(\eta^{*}\right)^{\prime} u_{2}\right\|_{L^{2}(Q)^{N}}^{2} \\
& +\left\|\left(\eta^{*}\right)^{\prime \prime} u_{2}\right\|_{L^{2}(Q)^{N}}^{2}+\left\|\eta^{*} u_{2, t}\right\|_{L^{2}(Q)^{N}}, \\
J_{4}\left(w_{2}\right)= & \left\|\left(\eta^{*}\right)^{\prime} \Delta w_{2}\right\|_{L^{2}(Q)^{N}}^{2}+\left\|\eta^{*} \Delta w_{2}\right\|_{L^{2}(Q)}^{2}+\left\|\left(\eta^{*}\right)^{\prime \prime} w_{2}\right\|_{L^{2}(Q)}^{2} \\
& +\left\|\left(\eta^{*}\right)^{\prime} w_{2, t}\right\|_{L^{2}(Q)}^{2}+\left\|\eta^{*} w_{2, t}\right\|_{L^{2}(Q)}^{2} .
\end{aligned}
$$

\subsubsection{Last arrangements and conclusion}

From the definition of $\left(u_{2}, w_{2}\right)=\left(-u_{1}+\eta \varphi,-w_{1}+\eta \psi\right)$, we can estimate $J_{3}\left(u_{2}\right)$ and $J_{4}\left(w_{2}\right)$ by

$$
\begin{aligned}
& \left\|\eta^{*} \Delta u_{1}\right\|_{L^{2}(Q)^{N}}^{2}+\left\|\left(\eta^{*}\right)^{\prime} \Delta u_{1}\right\|_{L^{2}(Q)^{N}}^{2}+\left\|\left(\eta^{*}\right)^{\prime} u_{1, t}\right\|_{L^{2}(Q)^{N}}^{2}+\left\|\left(\eta^{*}\right)^{\prime} u_{1}\right\|_{L^{2}(Q)^{N}}^{2}+\left\|\left(\eta^{*}\right)^{\prime \prime} u_{1}\right\|_{L^{2}(Q)^{N}}^{2} \\
& \quad+\left\|\eta^{*} u_{1, t}\right\|_{L^{2}(Q)^{N}}^{2}+\left\|\eta^{*} \eta \Delta \varphi\right\|_{L^{2}(Q)^{N}}^{2}+\left\|\left(\eta^{*}\right)^{\prime} \eta \Delta \varphi\right\|_{L^{2}(Q)^{N}}^{2}+\left\|\left(\eta^{*}\right)^{\prime}(\eta \varphi)_{t}\right\|_{L^{2}(Q)^{N}}^{2} \\
& \quad+\left\|\left(\eta^{*}\right)^{\prime} \eta \varphi\right\|_{L^{2}(Q)^{N}}^{2}+\left\|\left(\eta^{*}\right)^{\prime \prime} \eta \varphi\right\|_{L^{2}(Q)^{N}}^{2}+\left\|\eta^{*}(\eta \varphi)_{t}\right\|_{L^{2}(Q)^{N}}^{2}
\end{aligned}
$$

and

$$
\begin{aligned}
& \left\|\left(\eta^{*}\right)^{\prime} \Delta w_{1}\right\|_{L^{2}(Q)}^{2}+\left\|\eta^{*} \Delta w_{1}\right\|_{L^{2}(Q)}^{2}+\left\|\left(\eta^{*}\right)^{\prime \prime} w_{1}\right\|_{L^{2}(Q)}^{2}+\left\|\left(\eta^{*}\right)^{\prime} w_{1, t}\right\|_{L^{2}(Q)}^{2}+\left\|\eta^{*} w_{1, t}\right\|_{L^{2}(Q)}^{2} \\
& \quad+\left\|\left(\eta^{*}\right)^{\prime} \eta \Delta \psi\right\|_{L^{2}(Q)}^{2}+\left\|\eta^{*} \eta \Delta \psi\right\|_{L^{2}(Q)}^{2}+\left\|\left(\eta^{*}\right)^{\prime \prime} \eta \psi\right\|_{L^{2}(Q)}^{2}+\left\|\left(\eta^{*}\right)^{\prime}(\eta \psi)_{t}\right\|_{L^{2}(Q)}^{2}+\left\|\eta^{*}(\eta \psi)_{t}\right\|_{L^{2}(Q)}^{2},
\end{aligned}
$$


respectively.

For all the terms concerning $u_{1}$ and $w_{1}$, we can use estimate (36) since $\eta^{*},\left(\eta^{*}\right)^{\prime}$ and $\left(\eta^{*}\right)^{\prime \prime}$ are bounded functions in $(0, T)$ for $s \geqslant C\left(T^{4}+T^{8}\right)$. Hence, we have from (52)-(55)

$$
\begin{aligned}
s^{2} \lambda^{2} \int_{\omega_{2} \times(0, T)} \mathrm{e}^{-2 s \hat{\alpha} \hat{\xi}^{2}\left|\varphi_{t}\right|^{2} \mathrm{~d} x \mathrm{~d} t \leqslant} & C\left(1+\|\bar{y}\|_{\infty}^{8}+\|\bar{\theta}\|_{\infty}^{8}\right)\left\|\left(\bar{y}_{t}, \bar{\theta}_{t}\right)\right\|_{L^{2}\left(0, T ; L^{r}(\Omega)^{N+1}\right)}^{2} \mathrm{e}^{C T\left(1+\|\bar{y}\|_{\infty}^{2}+\|\bar{\theta}\|_{\infty}^{2}\right)} \\
& \times\left(\lambda ^ { 1 4 } ( 1 + T ) \left(\left\|\eta g_{1}\right\|_{L^{2}(Q)^{N}}^{2}+\left\|\eta g_{2}\right\|_{L^{2}(Q)}^{2}+\|\eta \varphi\|_{L^{2}\left(0, T ; L^{2}\left(\omega_{5}\right)^{N}\right)}^{2}\right.\right. \\
& +\|\eta \nabla \varphi\|_{L^{2}\left(0, T ; L^{2}\left(\omega_{5}\right)^{N}\right)}^{2}+\left\|\eta^{\prime} \varphi\right\|_{L^{2}\left(0, T ; L^{2}\left(\omega_{5}\right)^{N}\right)}^{2} \\
& \left.+\|\eta \nabla \psi\|_{L^{2}\left(0, T ; L^{2}\left(\omega_{5}\right)^{N}\right)}^{2}\right)+\left(1+T^{1 / 2}\right)\left(\left\|\eta^{*} \eta^{\prime \prime} \varphi\right\|_{L^{2}(Q)^{N}}^{2}\right. \\
& +\left\|\left(\eta^{*}\right)^{\prime} \eta^{\prime} \varphi\right\|_{L^{2}(Q)^{N}}^{2}+\left\|\eta^{*} \eta^{\prime} \varphi_{t}\right\|_{L^{2}(Q)^{N}}^{2}+\left\|\eta^{*} \eta \Delta \varphi\right\|_{L^{2}(Q)^{N}}^{2} \\
& +\left\|\left(\eta^{*}\right)^{\prime} \eta \Delta \varphi\right\|_{L^{2}(Q)^{N}}^{2}+\left\|\left(\eta^{*}\right)^{\prime} \eta \varphi_{t}\right\|_{L^{2}(Q)^{N}}^{2}+\left\|\left(\eta^{*}\right)^{\prime} \eta \varphi\right\|_{L^{2}(Q)^{N}}^{2} \\
& +\left\|\left(\eta^{*}\right)^{\prime \prime} \eta \varphi\right\|_{L^{2}(Q)^{N}}^{2}+\left\|\eta^{*} \eta^{\prime} \varphi\right\|_{L^{2}(Q)^{N}}^{2}+\left\|\eta^{*} \eta \varphi_{t}\right\|_{L^{2}(Q)^{N}}^{2} \\
& +\left\|\eta^{*} \eta^{\prime} \Delta \varphi\right\|_{L^{2}(Q)^{N}}^{2}+\left\|\left(\eta^{*}\right)^{\prime} \eta \Delta \psi\right\|_{L^{2}(Q)^{N}}^{2}+\left\|\eta^{*} \eta \Delta \psi\right\|_{L^{2}(Q)}^{2} \\
& +\left\|\left(\eta^{*}\right)^{\prime} \eta^{\prime} \psi\right\|_{L^{2}(Q)}^{2}+\left\|\left(\eta^{*}\right)^{\prime \prime} \eta \psi\right\|_{L^{2}(Q)}^{2}+\left\|\left(\eta^{*}\right)^{\prime} \eta \psi_{t}\right\|_{L^{2}(Q)}^{2} \\
& +\left\|\eta^{*} \eta^{\prime} \psi\right\|_{L^{2}(Q)}^{2}+\left\|\eta^{*} \eta \psi_{t}\right\|_{L^{2}(Q)}^{2}+\left\|\eta^{*} \eta^{\prime \prime} \psi\right\|_{L^{2}(Q)}^{2} \\
& +\left\|\eta^{*} \eta^{\prime} \psi_{t}\right\|_{L^{2}(Q)}^{2}+\left\|\eta^{*} \eta \varphi_{N, t}\right\|_{L^{2}(Q)}^{2}+\left\|\eta^{*} \eta^{\prime} \varphi_{N}\right\|_{L^{2}(Q)}^{2} \\
& \left.\left.+\left\|\eta^{*} \eta^{\prime} \Delta \psi\right\|_{L^{2}(Q)}+\left\|\left(\eta^{*}\right)^{\prime} \eta \varphi_{N}\right\|_{L^{2}(Q)}+\left\|\eta^{*} \eta \Delta \varphi_{N}\right\|_{L^{2}(Q)}\right)\right)
\end{aligned}
$$

Let us now estimate the global terms on $\varphi$ and $\psi$ and check that they can be eliminated using the left-hand side of (30). To this end, let us first write down some bounds of the weight functions appearing in (56):

$$
\begin{aligned}
& \left|\eta^{*} \eta\right| \leqslant C s^{-7 / 4} \lambda^{-5} \mathrm{e}^{-s \alpha^{*}} \hat{\xi}^{-7 / 4}, \\
& \left|\eta^{*} \eta^{\prime}\right|+\left|\left(\eta^{*}\right)^{\prime} \eta\right| \leqslant C T s^{-3 / 4} \lambda^{-5} \mathrm{e}^{-s \alpha^{*}} \hat{\xi}^{-1 / 2}, \\
& \left|\left(\eta^{*}\right)^{\prime} \eta^{\prime}\right|+\left|\left(\eta^{*}\right)^{\prime \prime} \eta\right|+\left|\eta^{*} \eta^{\prime \prime}\right| \leqslant C T^{2} s^{1 / 4} \lambda^{-5} \mathrm{e}^{-s \alpha^{*}}\left(\xi^{*}\right)^{3 / 4} .
\end{aligned}
$$

By virtue of these estimates, it is not difficult to see that, for all $0<\beta \leqslant 1 / 2$, we have

$$
T^{\beta}\left(\left|\eta^{*} \eta^{\prime}\right|+\left|\left(\eta^{*}\right)^{\prime} \eta\right|\right) \leqslant C s^{-1 / 2} \lambda^{-5} \mathrm{e}^{-s \alpha^{*}} \hat{\xi}^{-1 / 2}
$$

and

$$
T^{\beta}\left(\left|\left(\eta^{*}\right)^{\prime} \eta^{\prime}\right|+\left|\left(\eta^{*}\right)^{\prime \prime} \eta\right|+\left|\eta^{*} \eta^{\prime \prime}\right|\right) \leqslant C s^{3 / 2} \lambda^{-5} \mathrm{e}^{-s \alpha^{*}}\left(\xi^{*}\right)^{3 / 2}
$$

for $s \geqslant C\left(T^{4}+T^{8}\right)$.

Combining this and (56), we get

$$
\begin{aligned}
& s^{2} \lambda^{2} \int_{\omega_{2} \times(0, T)} \mathrm{e}^{-2 s \hat{\alpha}} \hat{\xi}^{2}\left|\varphi_{t}\right|^{2} \mathrm{~d} x \mathrm{~d} t \leqslant C\left(1+\|\bar{y}\|_{\infty}^{8}+\|\bar{\theta}\|_{\infty}^{8}\right)\left\|\left(\bar{y}_{t}, \bar{\theta}_{t}\right)\right\|_{L^{2}\left(0, T ; L^{r}(\Omega)^{N+1}\right)}^{2} \mathrm{e}^{C T\left(1+\|\bar{y}\|_{\infty}^{2}+\|\bar{\theta}\|_{\infty}^{2}\right)} \\
& \quad \times\left(\lambda ^ { 1 4 } ( 1 + T ) \left(\left\|\eta g_{1}\right\|_{L^{2}(Q)^{N}}^{2}+\left\|\eta g_{2}\right\|_{L^{2}(Q)}^{2}+\|\eta \varphi\|_{L^{2}\left(0, T ; L^{2}\left(\omega_{5}\right)^{N}\right)}^{2}\right.\right. \\
& \left.\quad+\|\eta \nabla \varphi\|_{L^{2}\left(0, T ; L^{2}\left(\omega_{5}\right)^{N}\right)}^{2}+\left\|\eta^{\prime} \varphi\right\|_{L^{2}\left(0, T ; L^{2}\left(\omega_{5}\right)^{N}\right)}^{2}+\|\eta \nabla \psi\|_{L^{2}\left(0, T ; L^{2}\left(\omega_{5}\right)^{N}\right)}^{2}\right)
\end{aligned}
$$




$$
\begin{aligned}
& +s^{3} \lambda^{-10} \iint_{Q} \mathrm{e}^{-2 s \alpha^{*}}\left(\xi^{*}\right)^{3}\left(|\varphi|^{2}+|\psi|^{2}\right) \mathrm{d} x \mathrm{~d} t \\
& \left.+s^{-1} \lambda^{-10} \iint_{Q} \mathrm{e}^{-2 s \alpha^{*}} \hat{\xi}^{-1}\left(\left|\varphi_{t}\right|^{2}+|\Delta \varphi|^{2}+\left|\psi_{t}\right|^{2}+|\Delta \psi|^{2}\right) \mathrm{d} x \mathrm{~d} t\right)
\end{aligned}
$$

for $s \geqslant C\left(T^{4}+T^{8}\right)$.

As $\alpha^{*}(t)=\max _{x \in \bar{\Omega}} \alpha(x, t)$, taking

$$
\lambda \geqslant C\left(1+\|\bar{y}\|_{\infty}+\|\bar{\theta}\|_{\infty}+\left\|\bar{y}_{t}\right\|_{L^{2}\left(0, T ; L^{r}(\Omega)^{N}\right)}^{2}+\left\|\bar{\theta}_{t}\right\|_{L^{2}\left(0, T ; L^{r}(\Omega)\right)}^{2}+\mathrm{e}^{C T\left(1+\|\bar{y}\|_{\infty}^{2}+\|\bar{\theta}\|_{\infty}^{2}\right)}\right),
$$

we obtain

$$
\begin{aligned}
& s^{2} \lambda^{2} \iint_{\omega_{2} \times(0, T)} \mathrm{e}^{-2 s \hat{\alpha}} \hat{\xi}^{2}\left|\varphi_{t}\right|^{2} \mathrm{~d} x \mathrm{~d} t \leqslant C^{*} \lambda^{24}(1+T)\left(\left\|\eta g_{1}\right\|_{L^{2}(Q)^{N}}^{2}+\left\|\eta g_{2}\right\|_{L^{2}(Q)}^{2}+\|\eta \varphi\|_{L^{2}\left(0, T ; L^{2}\left(\omega_{5}\right)^{N}\right)}^{2}\right. \\
& \left.+\left\|\eta^{\prime} \varphi\right\|_{L^{2}\left(0, T ; L^{2}\left(\omega_{5}\right)^{N}\right)}^{2}+\|\eta \nabla \varphi\|_{L^{2}\left(0, T ; L^{2}\left(\omega_{5}\right)^{N}\right)}^{2}+\|\eta \nabla \psi\|_{L^{2}\left(0, T ; L^{2}\left(\omega_{5}\right)^{N}\right)}^{2}\right) \\
& +\varepsilon(I(s, \lambda ; \varphi)+I(s, \lambda ; \psi)),
\end{aligned}
$$

for a positive constant $C^{*}$. Here, $\varepsilon>0$ is a small constant which depends on $\Omega$ and $\omega$.

Let us finally combine this inequality, (30), (31) and the Appendix. We find:

$$
\begin{aligned}
I(s, \lambda ; \varphi)+I(s, \lambda ; \psi) \leqslant & C \lambda^{24}(1+T)\left(\left\|\eta g_{1}\right\|_{L^{2}(Q)^{N}}^{2}+\left\|\eta g_{2}\right\|_{L^{2}(Q)}^{2}\right. \\
& +\|\eta \varphi\|_{L^{2}\left(0, T ; L^{2}\left(\omega_{5}\right)^{N}\right)}^{2}+\left\|\eta^{\prime} \varphi\right\|_{L^{2}\left(0, T ; L^{2}\left(\omega_{5}\right)^{N}\right)}^{2}+\|\eta \nabla \varphi\|_{L^{2}\left(0, T ; L^{2}\left(\omega_{5}\right)^{N}\right)}^{2} \\
& \left.+\|\eta \psi\|_{L^{2}\left(0, T ; L^{2}\left(\omega_{5}\right)\right)}^{2}+\|\eta \nabla \psi\|_{L^{2}\left(0, T ; L^{2}\left(\omega_{5}\right)^{N}\right)}^{2}\right)
\end{aligned}
$$

for $s \geqslant C\left(T^{4}+T^{8}\right)$ and

$$
\lambda \geqslant C\left(1+\|\bar{y}\|_{\infty}+\|\bar{\theta}\|_{\infty}+\left\|\bar{y}_{t}\right\|_{L^{2}\left(0, T ; L^{r}(\Omega)^{N}\right)}^{2}+\left\|\bar{\theta}_{t}\right\|_{L^{2}\left(0, T ; L^{r}(\Omega)\right)}^{2}+\mathrm{e}^{C T\left(1+\|\bar{y}\|_{\infty}^{2}+\|\bar{\theta}\|_{\infty}^{2}\right)}\right) .
$$

It only remains to get rid of the local terms on $\nabla \varphi$ and $\nabla \psi$ in the right-hand side of (57). Thus, let us introduce a cut-off function $\zeta \in C_{c}^{2}(\omega)$ such that

$$
\zeta \equiv 1 \quad \text { in } \omega_{5} \text { and } 0 \leqslant \zeta \leqslant 1 \quad \text { in } \omega .
$$

Then we have

$$
\begin{aligned}
\int_{\omega_{5} \times(0, T)}|\eta|^{2}|\nabla \varphi|^{2} \mathrm{~d} x \mathrm{~d} t & \leqslant \int_{\omega \times(0, T)}|\eta|^{2} \zeta|\nabla \varphi|^{2} \mathrm{~d} x \mathrm{~d} t \\
& =\frac{1}{2} \iint_{\omega \times(0, T)}|\eta|^{2} \Delta \zeta|\varphi|^{2} \mathrm{~d} x \mathrm{~d} t-\iint_{\omega \times(0, T)}|\eta|^{2} \zeta \Delta \varphi \cdot \varphi \mathrm{d} x \mathrm{~d} t .
\end{aligned}
$$

Let us apply Young's inequality to the last integral. This gives

$$
\begin{aligned}
\int_{\omega 5 \times(0, T)}|\eta|^{2}|\nabla \varphi|^{2} \mathrm{~d} x \mathrm{~d} t \leqslant & C s \lambda^{24}(1+T) \iint_{\omega \times(0, T)} \mathrm{e}^{2 s \alpha^{*}}|\eta|^{4} \hat{\xi}|\varphi|^{2} \mathrm{~d} x \mathrm{~d} t \\
& +\frac{1}{2 C^{*}} s^{-1} \lambda^{-24}(1+T)^{-1} \iint_{\omega \times(0, T)} \mathrm{e}^{-2 s \alpha^{*} \hat{\xi}^{-1}|\Delta \varphi|^{2} \mathrm{~d} x \mathrm{~d} t}
\end{aligned}
$$


for a constant $C(\Omega, \omega)>0$. Analogous computation can be done for $\nabla \psi$ and these would give exactly the same estimate.

Consequently, for any

$$
\lambda \geqslant C\left(1+\|\bar{y}\|_{\infty}+\|\bar{\theta}\|_{\infty}+\left\|\bar{y}_{t}\right\|_{L^{2}\left(0, T ; L^{r}(\Omega)^{N}\right)}^{2}+\left\|\bar{\theta}_{t}\right\|_{L^{2}\left(0, T ; L^{r}(\Omega)\right)}^{2}+\mathrm{e}^{C T\left(1+\|\bar{y}\|_{\infty}^{2}+\|\bar{\theta}\|_{\infty}^{2}\right)}\right),
$$

and $s \geqslant C\left(T^{4}+T^{8}\right)$, we obtain from (57)

$$
\begin{aligned}
I(s, \lambda ; \varphi)+I(s, \lambda ; \psi) \leqslant & C\left(1+T^{2}\right)\left(s^{15 / 2} \lambda^{24} \iint_{Q} \mathrm{e}^{-4 s \hat{\alpha}+2 s \alpha^{*}} \hat{\xi}^{15 / 2}\left(\left|g_{1}\right|^{2}+\left|g_{2}\right|^{2}\right) \mathrm{d} x \mathrm{~d} t\right. \\
& \left.+s^{16} \lambda^{48} \iint_{\omega \times(0, T)} \mathrm{e}^{-8 s \hat{\alpha}+6 s \alpha^{*}} \hat{\xi}^{16}\left(|\varphi|^{2}+|\psi|^{2}\right) \mathrm{d} x \mathrm{~d} t\right),
\end{aligned}
$$

which is exactly (17).

\section{Null controllability of (13)}

In this section, we will prove the null controllability of the following system:

$$
\begin{cases}L_{1} z+\nabla q=f_{1}+\rho e_{N}+v_{1} 1_{\omega} & \text { in } Q, \\ L_{2} \rho+\nabla \cdot(\bar{\theta} z)=f_{2}+v_{2} 1_{\omega} & \text { in } Q, \\ \nabla \cdot z=0 & \text { in } Q, \\ z=0, \quad \rho=0 & \text { on } \Sigma, \\ z(0)=z^{0}, \quad \rho(0)=\rho^{0} & \text { in } \Omega,\end{cases}
$$

where

$$
L_{1} z=z_{t}-\Delta z+(\bar{y} \cdot \nabla) z+(z \cdot \nabla) \bar{y}
$$

and

$$
L_{2} \rho=\rho_{t}-\Delta \rho+\bar{y} \cdot \nabla \rho .
$$

Before proving this, we will deduce a Carleman inequality with weight functions that do not vanish at $t=0$. More precisely, let us consider the function

$$
\ell(t)= \begin{cases}T^{2} / 4 & \text { for } 0 \leqslant t \leqslant T / 2 \\ t(T-t) & \text { for } T / 2 \leqslant t \leqslant T\end{cases}
$$

and the following associated weight functions:

$$
\begin{aligned}
& \beta(x, t)=\frac{\mathrm{e}^{5 / 4 \lambda m\left\|\eta^{0}\right\|_{\infty}}-\mathrm{e}^{\lambda\left(m\left\|\eta^{0}\right\|_{\infty}+\eta^{0}(x)\right)}}{\ell(t)^{4}}, \\
& \hat{\beta}(t)=\min _{x \in \bar{\Omega}} \beta(x, t), \quad \beta^{*}(t)=\max _{x \in \bar{\Omega}} \beta(x, t), \\
& \gamma(x, t)=\frac{\mathrm{e}^{\lambda\left(m\left\|\eta^{0}\right\|_{\infty}+\eta^{0}(x)\right)}}{\ell(t)^{4}}, \\
& \hat{\gamma}(t)=\max _{x \in \bar{\Omega}} \gamma(x, t), \quad \gamma^{*}(t)=\min _{x \in \bar{\Omega}} \gamma(x, t) .
\end{aligned}
$$


Lemma 2. Let $(\bar{y}, \bar{\theta})$ verify (5). Then, there exists a positive constant $C$ depending on $T, s, \lambda, \bar{y}$ and $\bar{\theta}$ such that every solution to the adjoint system (14) verifies

$$
\begin{aligned}
& \|\varphi(0)\|_{L^{2}(\Omega)^{N}}^{2}+\|\psi(0)\|_{L^{2}(\Omega)}^{2}+\iint_{Q} \mathrm{e}^{-2 s \beta} \gamma^{3}\left(|\varphi|^{2}+|\psi|^{2}\right) \mathrm{d} x \mathrm{~d} t+\iint_{Q} \mathrm{e}^{-2 s \beta} \gamma\left(|\nabla \varphi|^{2}+|\nabla \psi|^{2}\right) \mathrm{d} x \mathrm{~d} t \\
& \quad \leqslant C\left(\iint_{\omega \times(0, T)} \mathrm{e}^{-8 s \hat{\beta}+6 s \beta^{*}} \hat{\gamma}^{16}\left(|\varphi|^{2}+|\psi|^{2}\right) \mathrm{d} x \mathrm{~d} t+\iint_{Q} \mathrm{e}^{-4 s \hat{\beta}+2 s \beta^{*}} \hat{\gamma}^{15 / 2}\left(\left|g_{1}\right|^{2}+\left|g_{2}\right|^{2}\right) \mathrm{d} x \mathrm{~d} t\right) .
\end{aligned}
$$

Proof. From Carleman inequality (17), the proof of this lemma is classical. The main tool used is the dissipativity of system (14). Observe that this tells us that $\left(\|\varphi(t)\|_{L^{2}(\Omega)^{N}},\|\psi(t)\|_{L^{2}(\Omega)}\right)$ are 'increasing' functions of $t$, so the presence of $\left(\|\varphi(0)\|_{L^{2}(\Omega)^{N}},\|\psi(0)\|_{L^{2}(\Omega)}\right)$ in the left-hand side of (61) is not a surprise. Anyway, we decide to include the proof here for completeness (for more details see, for instance, [4]).

We start with an a priori estimate for the Boussinesq system (14):

$$
\begin{aligned}
& \|\varphi\|_{L^{2}(0, T / 2 ; V)}+\|\varphi\|_{L^{\infty}(0, T / 2 ; H)}+\|\psi\|_{L^{2}\left(0, T / 2 ; H^{1}(\Omega)\right)}+\|\psi\|_{L^{\infty}\left(0, T / 2 ; L^{2}(\Omega)\right)} \\
& \leqslant \\
& \quad C\left(\left\|g_{1}\right\|_{L^{2}\left(0,3 T / 4 ; L^{2}(\Omega)^{N}\right)}+\|\varphi\|_{L^{2}\left(T / 2,3 T / 4 ; L^{2}(\Omega)^{N}\right)}\right. \\
& \left.\quad+\left\|g_{2}\right\|_{L^{2}\left(0,3 T / 4 ; L^{2}(\Omega)\right)}+\|\psi\|_{L^{2}\left(T / 2,3 T / 4 ; L^{2}(\Omega)\right)}\right),
\end{aligned}
$$

for a positive constant $C$ depending on $\Omega, T,\|\bar{y}\|_{\infty}$ and $\|\bar{\theta}\|_{\infty}$. To prove this, it suffices to combine the energy estimates for $\gamma \varphi$ and $\gamma \psi$, where $\gamma \in C^{1}([0, T])$ satisfies

$$
\gamma \equiv 1 \quad \text { in }[0, T / 2] \quad \text { and } \quad \gamma \equiv 0 \quad \text { in }[3 T / 4, T] .
$$

Consequently, we can obtain a first estimate in $\Omega \times(0, T / 2)$ :

$$
\begin{aligned}
& \|\varphi(0)\|_{L^{2}(\Omega)^{N}}^{2}+\|\psi(0)\|_{L^{2}(\Omega)}^{2}+\int_{0}^{T / 2} \int_{\Omega} \mathrm{e}^{-2 s \beta} \gamma^{3}\left(|\varphi|^{2}+|\psi|^{2}\right) \mathrm{d} x \mathrm{~d} t+\int_{0}^{T / 2} \int_{\Omega} \mathrm{e}^{-2 s \beta} \gamma\left(|\nabla \varphi|^{2}+|\nabla \psi|^{2}\right) \mathrm{d} x \mathrm{~d} t \\
& \quad \leqslant C\left(\int_{0}^{3 T / 4} \int_{\Omega} \mathrm{e}^{-4 s \hat{\beta}+2 s \beta^{*}} \hat{\gamma}^{15 / 2}\left(\left|g_{1}\right|^{2}+\left|g_{2}\right|^{2}\right) \mathrm{d} x \mathrm{~d} t+\int_{T / 2}^{3 T / 4} \int_{\Omega} \mathrm{e}^{-2 s \beta} \gamma^{3}\left(|\varphi|^{2}+|\psi|^{2}\right) \mathrm{d} x \mathrm{~d} t\right) .
\end{aligned}
$$

On the other hand, since $\alpha=\beta$ in $\Omega \times(T / 2, T)$, we have

$$
\begin{aligned}
& \int_{T / 2}^{T} \int_{\Omega} \mathrm{e}^{-2 s \beta} \gamma^{3}\left(|\varphi|^{2}+|\psi|^{2}\right) \mathrm{d} x \mathrm{~d} t+\int_{T / 2}^{T} \int_{\Omega} \mathrm{e}^{-2 s \beta} \gamma\left(|\nabla \varphi|^{2}+|\nabla \psi|^{2}\right) \mathrm{d} x \mathrm{~d} t \\
& \quad=\int_{T / 2} \int_{\Omega} \mathrm{e}^{-2 s \alpha} \xi^{3}\left(|\varphi|^{2}+|\psi|^{2}\right) \mathrm{d} x \mathrm{~d} t+\int_{T / 2}^{T} \int_{\Omega} \mathrm{e}^{-2 s \alpha} \xi\left(|\nabla \varphi|^{2}+|\nabla \psi|^{2}\right) \mathrm{d} x \mathrm{~d} t \\
& \quad \leqslant C(I(s, \lambda ; \varphi)+I(s, \lambda ; \psi))
\end{aligned}
$$

so, by virtue of the Carleman inequality (17), we have:

$$
\int_{T / 2}^{T} \int_{\Omega} \mathrm{e}^{-2 s \beta} \gamma^{3}\left(|\varphi|^{2}+|\psi|^{2}\right) \mathrm{d} x \mathrm{~d} t+\int_{T / 2}^{T} \int_{\Omega} \mathrm{e}^{-2 s \beta} \gamma\left(|\nabla \varphi|^{2}+|\nabla \psi|^{2}\right) \mathrm{d} x \mathrm{~d} t
$$




$$
\leqslant C\left(\iint_{Q} \mathrm{e}^{-4 s \hat{\alpha}+2 s \alpha^{*}} \hat{\xi}^{15 / 2}\left(\left|g_{1}\right|^{2}+\left|g_{2}\right|^{2}\right) \mathrm{d} x \mathrm{~d} t+\iint_{\omega \times(0, T)} \mathrm{e}^{-8 s \hat{\alpha}+6 s \alpha^{*} \hat{\xi}^{16}}\left(|\varphi|^{2}+|\psi|^{2}\right) \mathrm{d} x \mathrm{~d} t\right) .
$$

Finally, from the definition of $\beta, \hat{\beta}, \beta^{*}, \gamma$ and $\hat{\gamma}$, we get

$$
\begin{aligned}
& \int_{T / 2}^{T} \int_{\Omega} \mathrm{e}^{-2 s \beta} \gamma^{3}\left(|\varphi|^{2}+|\psi|^{2}\right) \mathrm{d} x \mathrm{~d} t+\int_{T / 2}^{T} \int_{\Omega} \mathrm{e}^{-2 s \beta} \gamma\left(|\nabla \varphi|^{2}+|\nabla \psi|^{2}\right) \mathrm{d} x \mathrm{~d} t \\
& \quad \leqslant C\left(\iint_{Q} \mathrm{e}^{-4 s \hat{\beta}+2 s \beta^{*}} \hat{\gamma}^{15 / 2}\left(\left|g_{1}\right|^{2}+\left|g_{2}\right|^{2}\right) \mathrm{d} x \mathrm{~d} t+\iint_{\omega \times(0, T)} \mathrm{e}^{-8 s \hat{\beta}+6 s \beta^{*}} \hat{\gamma}^{16}\left(|\varphi|^{2}+|\psi|^{2}\right) \mathrm{d} x \mathrm{~d} t\right)
\end{aligned}
$$

which, together with (63), provides the desired inequality (61).

After having proved (61), we are ready to solve the null controllability problem for the linearized system (58). For simplicity, we introduce the following weight functions:

$$
\beta_{1}(t)=\mathrm{e}^{2 s \hat{\beta}-s \beta^{*}} \hat{\gamma}^{-15 / 4}(t), \quad \beta_{2}(t)=\mathrm{e}^{4 s \hat{\beta}-3 s \beta^{*}} \hat{\gamma}^{-8}(t)
$$

and

$$
\beta_{3}(t)=\mathrm{e}^{s \beta^{*}}\left(\gamma^{*}\right)^{-1 / 2}(t)
$$

In order to apply a inverse mapping theorem argument to the nonlinear problem (11) (Section 4), we will have to solve the null controllability of (58) with $(z, q, \rho)$ belonging to a suitable weighted space, which we present now:

$$
\begin{aligned}
& \widetilde{E}_{2}=\left\{\left(z, q, v_{1}, \rho, v_{2}\right):\left(z, v_{1}, \rho, v_{2}\right) \in \widetilde{E}_{0}, \beta_{3}\left(L_{1} z+\nabla q-\rho e_{N}-v_{1} 1_{\omega}\right) \in L^{2}\left(0, T ; H^{-1}(\Omega)^{2}\right),\right. \\
&\left.\beta_{3}\left(L_{2} \rho+\nabla \cdot(\bar{\theta} z)-v_{2} 1_{\omega}\right) \in L^{2}\left(0, T ; H^{-1}(\Omega)\right)\right\}
\end{aligned}
$$

when $N=2$ and

$$
\begin{aligned}
\widetilde{E}_{3}=\{ & \left(z, q, v_{1}, \rho, v_{2}\right):\left(z, v_{1}, \rho, v_{2}\right) \in \widetilde{E}_{0}, \beta_{3}^{1 / 2} z \in L^{4}\left(0, T ; L^{12}(\Omega)^{3}\right), \\
& \beta_{3}\left(L_{1} z+\nabla q-\rho e_{N}-v_{1} 1_{\omega}\right) \in L^{2}\left(0, T ; W^{-1,6}(\Omega)^{3}\right), \\
& \left.\beta_{3}\left(L_{2} \rho+\nabla \cdot(\bar{\theta} z)-v_{2} 1_{\omega}\right) \in L^{2}\left(0, T ; H^{-1}(\Omega)\right)\right\}
\end{aligned}
$$

when $N=3$, where

$$
\begin{aligned}
& \widetilde{E}_{0}=\left\{\left(z, v_{1}, \rho, v_{2}\right):\left(\beta_{1} z\right)_{i}, \beta_{1} \rho, \beta_{2}\left(v_{1} 1_{\omega}\right)_{i}, \beta_{2} v_{2} 1_{\omega} \in L^{2}(Q), \beta_{3}^{1 / 2} z \in L^{2}(0, T ; V) \cap L^{\infty}(0, T ; H),\right. \\
&\left.\beta_{3}^{1 / 2} \rho \in L^{2}\left(0, T ; H^{1}(\Omega)\right) \cap L^{\infty}\left(0, T ; L^{2}(\Omega)\right)\right\} .
\end{aligned}
$$

Of course, $\widetilde{E}_{2}$ and $\widetilde{E}_{3}$ are Banach spaces for the norms

$$
\begin{aligned}
\left\|\left(z, q, v_{1}, \rho, v_{2}\right)\right\|_{\widetilde{E}_{2}}= & \left(\left\|\left(z, v_{1}, \rho, v_{2}\right)\right\|_{\widetilde{E}_{0}}^{2}+\left\|\beta_{3}\left(L_{1} z+\nabla q-\rho e_{N}-v_{1} 1_{\omega}\right)\right\|_{L^{2}\left(0, T ; H^{-1}(\Omega)^{2}\right)}^{2}\right. \\
& \left.+\left\|\beta_{3}\left(L_{2} \rho+\nabla \cdot(\bar{\theta} z)-v_{2} 1_{\omega}\right)\right\|_{L^{2}\left(0, T ; H^{-1}(\Omega)\right)}^{2}\right)^{1 / 2}
\end{aligned}
$$

and

$$
\begin{aligned}
\left\|\left(z, q, v_{1}, \rho, v_{2}\right)\right\|_{\widetilde{E}_{3}=} & \left(\left\|\left(z, v_{1}, \rho, v_{2}\right)\right\|_{\widetilde{E}_{0}}^{2}+\left\|\beta_{3}^{1 / 2} z\right\|_{L^{4}\left(0, T ; L^{12}(\Omega)^{3}\right)}^{2}\right. \\
& +\left\|\beta_{3}\left(L_{1} z+\nabla q-\rho e_{N}-v_{1} 1_{\omega}\right)\right\|_{L^{2}\left(0, T ; W^{-1,6}(\Omega)^{3}\right)}^{2} \\
& \left.+\left\|\beta_{3}\left(L_{2} \rho+\nabla \cdot(\bar{\theta} z)-v_{2} 1_{\omega}\right)\right\|_{L^{2}\left(0, T ; H^{-1}(\Omega)\right)}^{2}\right)^{1 / 2}
\end{aligned}
$$


where

$$
\begin{aligned}
\left\|\left(z, v_{1}, \rho, v_{2}\right)\right\|_{\widetilde{E}_{0}=} & \left(\left\|\beta_{1} z\right\|_{L^{2}(Q)^{N}}^{2}+\left\|\beta_{1} \rho\right\|_{L^{2}(Q)}^{2}+\left\|\beta_{2} v_{1} 1_{\omega}\right\|_{L^{2}(Q)^{N}}^{2}\right. \\
& +\left\|\beta_{2} v_{2} 1_{\omega}\right\|_{L^{2}(Q)}^{2}+\left\|\beta_{3}^{1 / 2} z\right\|_{L^{2}(0, T ; V) \cap L^{\infty}(0, T ; H)}^{2} \\
& \left.+\left\|\beta_{3}^{1 / 2} \rho\right\|_{L^{2}\left(0, T ; H^{1}(\Omega)\right) \cap L^{\infty}\left(0, T ; L^{2}(\Omega)\right)}^{2}\right)^{1 / 2} .
\end{aligned}
$$

Then, we have the following result:

Proposition 2. Let us assume that $(\bar{y}, \bar{\theta})$ satisfy (5) and the following hypotheses on the initial condition and the right-hand sides hold:

- If $N=2: z^{0} \in H, \rho^{0} \in L^{2}(\Omega), \beta_{3} f_{1} \in L^{2}\left(0, T ; H^{-1}(\Omega)^{2}\right)$ and $\beta_{3} f_{2} \in L^{2}\left(0, T ; H^{-1}(\Omega)\right)$.

- If $N=3: z^{0} \in H \cap L^{4}(\Omega)^{3}, \rho^{0} \in L^{2}(\Omega), \beta_{3} f_{1} \in L^{2}\left(0, T ; W^{-1,6}(\Omega)^{3}\right)$ and $\beta_{3} f_{2} \in L^{2}\left(0, T ; H^{-1}(\Omega)\right)$.

Then, there exist two controls $v_{1} \in L^{2}(\omega \times(0, T))^{N}$ and $v_{2} \in L^{2}(\underset{\widetilde{\omega}}{\omega} \times(0, T))$ such that, if $(z, \rho)$ is (together with some $q)$ the associated solution to (58), one has $\left(z, q, v_{1}, \rho, v_{2}\right) \in \widetilde{E}_{N}$. In particular, $z(T)=0$ and $\rho(T)=0$ in $\Omega$.

Proof. This proof follows the same steps as in [3] but adapted to this system. Consequently, we will may pass over some aspects of minor importance along the proof.

Let us introduce the extremal problem

$$
\begin{aligned}
& \inf \frac{1}{2}\left(\iint_{Q} \beta_{1}^{2}\left(|z|^{2}+|\rho|^{2}\right) \mathrm{d} x \mathrm{~d} t+\iint_{0} \beta_{2}^{2}\left(\left|v_{1}\right|^{2}+\left|v_{2}\right|^{2}\right) \mathrm{d} x \mathrm{~d} t\right) \\
& \text { subject to }\left(v_{1}\right)_{i}, v_{2} \in L^{2}(Q), \operatorname{supp} v_{1}, v_{2} \subset \omega \times(0, T) \text { and }
\end{aligned}
$$

Let us suppose for the moment that we have a solution $\left(\hat{z}, \hat{q}, \hat{v}_{1}, \hat{\rho}, \hat{v}_{2}\right)$ of (64). Then, in view of Lagrange's principle, there exist dual variables $\widehat{w}, \hat{h}$ and $\hat{\tau}$ such that

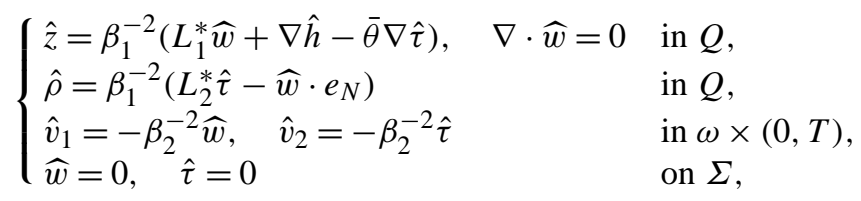

where $L_{i}^{*}$ is the adjoint operator of $L_{i}(i=1,2)$, i.e.

$$
L_{1}^{*} w=-w_{t}-\Delta w-D w \bar{y}
$$

and

$$
L_{2}^{*} \tau=-\tau_{t}-\Delta \tau-\bar{y} \cdot \nabla \tau .
$$

Let us now set

$$
P_{0}=\left\{(w, h, \tau) \in C^{\infty}(\bar{Q})^{N+2}: \nabla \cdot w=0 \text { in } Q,\left.w\right|_{\Sigma}=\left.\tau\right|_{\Sigma}=0, \int_{\omega} h(x, t) \mathrm{d} x=0\right\}
$$

and 


$$
\begin{aligned}
a((\widehat{w}, \hat{h}, \hat{\tau}),(w, h, \tau))= & \iint_{Q} \beta_{1}^{-2}\left(L_{1}^{*} \widehat{w}+\nabla \hat{h}-\bar{\theta} \nabla \hat{\tau}\right)\left(L_{1}^{*} w+\nabla h-\bar{\theta} \nabla \tau\right) \mathrm{d} x \mathrm{~d} t \\
& +\iint_{Q} \beta_{1}^{-2}\left(L_{2}^{*} \hat{\tau}-\widehat{w} \cdot e_{N}\right)\left(L_{2}^{*} \tau-w \cdot e_{N}\right) \mathrm{d} x \mathrm{~d} t \\
& +\iint_{\omega \times(0, T)} \beta_{2}^{-2}(\widehat{w} w+\hat{\tau} \tau) \mathrm{d} x \mathrm{~d} t \quad \forall(w, h, \tau) \in P_{0} .
\end{aligned}
$$

Then, we must have

$$
a((\widehat{w}, \hat{h}, \hat{\tau}),(w, h, \tau))=\left\langle\ell_{0},(w, h, \tau)\right\rangle \quad \forall(w, h, \tau) \in P_{0},
$$

where we have used the notation

$$
\begin{aligned}
\left\langle\ell_{0},(w, h, \tau)\right\rangle= & \int_{0}^{T}\left\langle f_{1}(t), w(t)\right\rangle_{H^{-1}(\Omega), H_{0}^{1}(\Omega)} \mathrm{d} t+\int_{0}^{T}\left\langle f_{2}(t), \tau(t)\right\rangle_{H^{-1}(\Omega), H_{0}^{1}(\Omega)} \mathrm{d} t \\
& +\int_{\Omega} z^{0} w(0) \mathrm{d} x+\int_{\Omega} \rho^{0} \tau(0) \mathrm{d} x .
\end{aligned}
$$

The next step is to demonstrate that there exists exactly one $(\widehat{w}, \hat{h}, \hat{\tau})$ satisfying (66) in an appropriate space. We will then define $\hat{z}, \hat{v}_{1}, \hat{\rho}$ and $\hat{v}_{2}$ using (65) and we will check that $\left(\hat{z}, \hat{v}_{1}, \hat{\rho}, \hat{v}_{2}\right)$ together with some $\hat{q}$, fulfills the desired properties.

Thus, consider the linear space $P_{0}$ and the bilinear form $a(\cdot, \cdot)$ on $P_{0}$ (which clearly is a scalar product) and observe that the Carleman inequality $(61)$ holds for all $(w, h, \tau) \in P_{0}$, i.e.

$$
\begin{aligned}
& \iint_{Q} \beta_{3}^{-2} \gamma^{2}\left(|w|^{2}+|\tau|^{2}\right) \mathrm{d} x \mathrm{~d} t+\iint_{Q} \beta_{3}^{-2}\left(|\nabla w|^{2}+|\nabla \tau|^{2}\right) \mathrm{d} x \mathrm{~d} t+\|w(0)\|_{L^{2}(\Omega)^{N}}^{2}+\|\tau(0)\|_{L^{2}(\Omega)}^{2} \\
& \quad \leqslant C a((w, h, \tau),(w, h, \tau)) \quad \forall(w, h, \tau) \in P_{0} .
\end{aligned}
$$

Let us now consider the space $P$, given by the completion of $P_{0}$ for the norm associated to $a(\cdot, \cdot)$ (which we denote by $\|\cdot\|_{P}$ ). This is a Hilbert space and $a(\cdot, \cdot)$ is a continuous and coercive bilinear form on $P$.

Let us also introduce the linear form $\ell_{0}$ given by $(67)$, for all $(w, h, \tau) \in P$. After a simple computation, we see that

$$
\begin{aligned}
\mid\left\langle\ell_{0},(w, h, \tau)\right\rangle \leqslant & \left\|\beta_{3} f_{1}\right\|_{L^{2}\left(0, T ; H^{-1}(\Omega)^{N}\right)}\left\|\beta_{3}^{-1} w\right\|_{L^{2}\left(0, T ; H_{0}^{1}(\Omega)^{N}\right)} \\
& +\left\|\beta_{3} f_{2}\right\|_{L^{2}\left(0, T ; H^{-1}(\Omega)\right)}\left\|\beta_{3}^{-1} \tau\right\|_{L^{2}\left(0, T ; H_{0}^{1}(\Omega)\right)} \\
& +\left\|z^{0}\right\|_{H}\|w(0)\|_{H}+\left\|\rho^{0}\right\|_{L^{2}(\Omega)}\|\tau(0)\|_{L^{2}(\Omega)} \quad \forall(w, h, \tau) \in P
\end{aligned}
$$

and, in particular, using (68) and the density of $P_{0}$ in $P$, we find:

$$
\left\{\begin{array}{c}
\left|\left\langle\ell_{0},(w, h, \tau)\right\rangle\right| \leqslant C\left(\left\|\beta_{3} f_{1}\right\|_{L^{2}\left(0, T ; H^{-1}(\Omega)^{N}\right)}+\left\|\beta_{3} f_{2}\right\|_{L^{2}\left(0, T ; H^{-1}(\Omega)\right)}\right. \\
\left.\quad+\left\|z^{0}\right\|_{H}+\left\|\rho^{0}\right\|_{L^{2}(\Omega)}\right)\|(w, h, \tau)\|_{P} \quad \forall(w, h, \tau) \in P .
\end{array}\right.
$$

In other words, $\ell_{0}$ is a bounded linear form on $P$. Consequently, in view of Lax-Milgram's lemma, there exists one and only one $(\widehat{w}, \hat{h}, \hat{\tau})$ satisfying

$$
\left\{\begin{array}{l}
a((\widehat{w}, \hat{h}, \hat{\tau}),(w, h, \tau))=\left\langle\ell_{0},(w, h, \tau)\right\rangle \quad \forall(w, h, \tau) \in P, \\
(\widehat{w}, \hat{h}, \tau) \in P .
\end{array}\right.
$$


Let us set

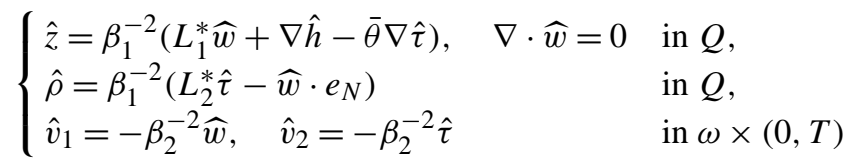

and let us see that $\left(\hat{z}, \hat{\rho}, \hat{v}_{1}, \hat{v}_{2}\right)$ verifies

$$
\iint_{Q} \beta_{1}^{2}\left(|\hat{z}|^{2}+|\hat{\rho}|^{2}\right) \mathrm{d} x \mathrm{~d} t+\iint_{\omega \times(0, T)} \beta_{2}^{2}\left(\left|\hat{v}_{1}\right|^{2}+\left|\hat{v}_{2}\right|^{2}\right) \mathrm{d} x \mathrm{~d} t<+\infty
$$

and is solution of the Boussinesq system in (64) for some pressure $\hat{q}$.

The first property is easy to check, since $(\widehat{w}, \hat{h}, \hat{\tau}) \in P$ and

$$
\iint_{Q} \beta_{1}^{2}\left(|\hat{z}|^{2}+|\hat{\rho}|^{2}\right) \mathrm{d} x \mathrm{~d} t+\iint_{\omega \times(0, T)} \beta_{2}^{2}\left(\left|\hat{v}_{1}\right|^{2}+\left|\hat{v}_{2}\right|^{2}\right) \mathrm{d} x \mathrm{~d} t=a((\widehat{w}, \hat{h}, \hat{\tau}),(\widehat{w}, \hat{h}, \hat{\tau})) .
$$

Notice that, in particular, $\hat{z} \in L^{2}(Q)^{N}, \hat{\rho} \in L^{2}(\Omega), \hat{v}_{1} \in L^{2}(\omega \times(0, T))^{N}$ and $\hat{v}_{2} \in L^{2}(\omega \times(0, T))$. Then, we introduce the (weak) solution $(\tilde{z}, \tilde{q}, \tilde{\rho})$ to the Boussinesq system

$$
\begin{cases}L_{1} \tilde{z}+\nabla \tilde{q}=f_{1}+\tilde{\rho} e_{N}+\hat{v}_{1} 1_{\omega}, \nabla \cdot \tilde{z}=0 & \text { in } Q, \\ L_{2} \tilde{\rho}+\nabla \cdot(\bar{\theta} \tilde{z})=f_{2}+\hat{v}_{2} 1_{\omega} & \text { in } Q, \\ \tilde{z}=0, \quad \tilde{\rho}=0 & \text { on } \Sigma, \\ \tilde{z}(0)=z^{0}, \quad \tilde{\rho}(0)=\rho^{0} & \text { in } \Omega .\end{cases}
$$

Clearly $(\tilde{z}, \tilde{\rho})$ is also, together with $\tilde{q}$, the unique solution of (71) defined by transposition. Of course, this means that $(\tilde{z}, \tilde{\rho})$ are the unique functions in $L^{2}(Q)^{N} \times L^{2}(Q)$ satisfying

$$
\left\{\begin{array}{l}
\iint_{Q} \tilde{z} \cdot b_{1} \mathrm{~d} x \mathrm{~d} t+\iint_{Q} \tilde{\rho} b_{2} \mathrm{~d} x \mathrm{~d} t=\int_{0}^{T}\left\langle f_{1}(t), w(t)\right\rangle_{H^{-1}(\Omega), H_{0}^{1}(\Omega)} \mathrm{d} t \\
\quad+\int_{0}^{T}\left\langle f_{2}(t), \tau(t)\right\rangle_{H^{-1}(\Omega), H_{0}^{1}(\Omega)} \mathrm{d} t+\iint_{Q}\left(\hat{v}_{1} 1_{\omega} \cdot w+\hat{v}_{2} 1_{\omega} \tau\right) \mathrm{d} x \mathrm{~d} t \\
\quad+\int_{\Omega} z^{0} \cdot w(0) \mathrm{d} x+\int_{\Omega} \rho^{0} \tau(0) \mathrm{d} x \quad \forall\left(b_{1}, b_{2}\right) \in L^{2}(Q)^{N} \times L^{2}(Q),
\end{array}\right.
$$

where $(w, \tau)$ is, together with some $h$, the solution to

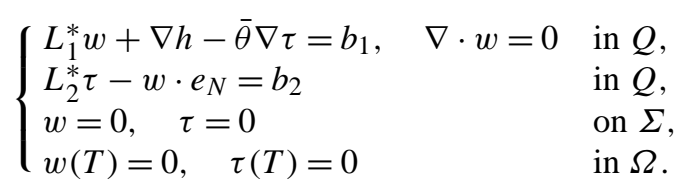

From (69) and (70), we see that $(\hat{z}, \hat{\rho})$ also satisfies (72). Consequently, $(\hat{z}, \hat{\rho})=(\tilde{z}, \tilde{\rho})$ and $(\hat{z}, \hat{\rho})$ is, together with $\hat{q}=\tilde{q}$, the solution to the Stokes system in (64).

Finally, we must see that $\left(\hat{z}, \hat{q}, \hat{\rho}, \hat{v}_{1}, \hat{v}_{2}\right) \in \widetilde{E}_{N}$. We already know that

$$
\begin{aligned}
& \left(\beta_{1} \hat{z}\right)_{i}, \beta_{1} \hat{\rho},\left(\beta_{2} \hat{v}_{1} 1_{\omega}\right)_{i}, \beta_{2} \hat{v}_{2} 1_{\omega} \in L^{2}(Q), \\
& \beta_{3}\left(L_{1} \hat{z}+\nabla \hat{q}-\hat{\rho} e_{N}-\hat{v}_{1} 1_{\omega}\right) \in \begin{cases}L^{2}\left(0, T ; H^{-1}(\Omega)^{2}\right) & \text { if } N=2, \\
L^{2}\left(0, T ; W^{-1,6}(\Omega)^{3}\right) & \text { if } N=3\end{cases}
\end{aligned}
$$


and

$$
\beta_{3}\left(L_{2} \hat{\rho}+\nabla \cdot(\bar{\theta} z)-\hat{v}_{2} 1_{\omega}\right) \in L^{2}\left(0, T ; H^{-1}(\Omega)\right) .
$$

Thus, it just remains to check that

$$
\beta_{3}^{1 / 2} \hat{z} \in L^{2}(0, T ; V) \cap L^{\infty}(0, T ; H), \quad \beta_{3}^{1 / 2} \hat{\rho} \in L^{2}\left(0, T ; H^{1}(\Omega)\right) \cap L^{\infty}\left(0, T ; L^{2}(\Omega)\right)
$$

and $\beta_{3}^{1 / 2} \hat{z} \in L^{4}\left(0, T ; L^{12}(\Omega)^{3}\right)$ in dimension 3. To this end, let us introduce the functions $z^{*}=\beta_{3}^{1 / 2} \hat{z}, q^{*}=\beta_{3}^{1 / 2} \hat{q}$, $\rho^{*}=\beta_{3}^{1 / 2} \rho, f_{1}^{*}=\beta_{3}^{1 / 2}\left(f_{1}+\hat{v}_{1} 1_{\omega}\right)$ and $f_{2}^{*}=\beta_{3}^{1 / 2}\left(f_{2}+\hat{v}_{2} 1_{\omega}\right)$. Then $\left(z^{*}, q^{*}, \rho^{*}\right)$ satisfies

$$
\begin{cases}L_{1} z^{*}+\nabla q^{*}=f_{1}^{*}+\rho^{*} e_{N}+\left(\beta_{3}^{1 / 2}\right)_{t} \hat{z}, \nabla \cdot z^{*}=0 & \text { in } Q, \\ L_{2} \rho^{*}+\nabla \cdot\left(\bar{\theta} z^{*}\right)=f_{2}^{*}+\left(\beta_{3}^{1 / 2}\right)_{t} \hat{\rho} & \text { in } Q, \\ z^{*}=0, \quad \rho^{*}=0 & \text { on } \Sigma, \\ z^{*}(0)=\beta_{3}^{1 / 2}(0) z^{0}, \quad \rho^{*}(0)=\beta_{3}^{1 / 2}(0) \rho^{0} & \text { in } \Omega .\end{cases}
$$

Since $\left(f_{1}^{*}\right)_{i}, f_{2}^{*} \in L^{2}\left(0, T ; H^{-1}(\Omega)\right),\left(\left(\beta_{3}^{1 / 2}\right)_{t} \hat{z}\right)_{i},\left(\beta_{3}^{1 / 2}\right)_{t} \hat{\rho} \in L^{2}(Q), z^{0} \in H$ and $\rho^{0} \in L^{2}(\Omega)$, we have

$$
z^{*} \in L^{2}(0, T ; V) \cap L^{\infty}(0, T ; H) \quad \text { and } \quad \rho^{*} \in L^{2}\left(0, T ; H^{1}(\Omega)\right) \cap L^{\infty}\left(0, T ; L^{2}(\Omega)\right) .
$$

Our last task will be to deduce that $z^{*} \in L^{4}\left(0, T ; L^{12}(\Omega)^{3}\right)$ when $N=3$. To this end, let us consider, for each $b \in L^{4 / 3}\left(0, T ; L^{12 / 11}(\Omega)^{3}\right)$, the Stokes system

$$
\begin{cases}-w_{t}-\Delta w+\nabla h=b & \text { in } Q, \\ \nabla \cdot w=0 & \text { in } Q, \\ w=0 & \text { on } \Sigma, \\ w(T)=0 & \text { in } \Omega .\end{cases}
$$

Under this assumption, it was proved in [3] (see Lemma 2) that

$$
w \in L^{2}\left(0, T ; W_{0}^{1,6 / 5}(\Omega)^{3}\right) \cap C^{0}\left([0, T] ; L^{4 / 3}(\Omega)^{3}\right),
$$

depending continuously on $b$ in these spaces.

Then $z^{*}$ must coincide with the solution by transposition of the first equation in (73), namely, the unique function $z^{*} \in L^{4}\left(0, T ; L^{12}(\Omega)^{3}\right)$ satisfying

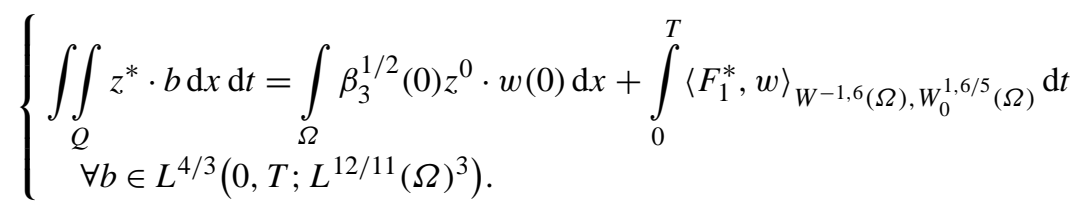

Here, $F_{1}^{*}$ stands for the function

$$
F_{1}^{*}=f_{1}^{*}+\left(\beta_{3}^{1 / 2}\right)_{t} \hat{z}+\rho^{*} e_{N}-\left(z^{*}, \nabla\right) \bar{y}-(\bar{y}, \nabla) z^{*}
$$

and $(w, h)$ is the solution of (74) associated to $b$. Remark that, as we already had that $z^{*} \in L^{2}\left(0, T ; L^{6}(\Omega)^{3}\right)$ and $\rho^{*} \in L^{2}(Q)$, all the terms of the previous definition make sense by virtue of (75) and the assumption $z^{0} \in L^{4}(\Omega)^{3}$.

Therefore, $z^{*} \in L^{4}\left(0, T ; L^{12}(\Omega)^{3}\right)$. This ends the proof of Proposition 2.

Remark 4. Observe that, besides the null controllability, we have got two additional (and essential) properties for the solution $(z, \rho)$ of $(58)$ :

- On the one hand, we have found $(z, \rho)$ decreasing exponentially as $t \rightarrow T^{-}$.

- On the other hand, a supplementary property has been obtained as indicates the $L^{4}\left(L^{12}\right)$ regularity for $z$. 


\section{Local null controllability of (11)}

In this section we establish the result that allows us to conclude Theorem 1, i.e., the local null controllability of system (11):

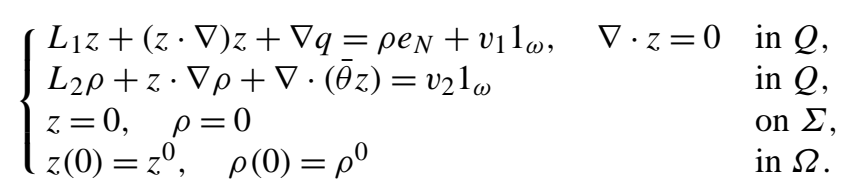

For this, we will follow the steps developed in [3]. Thus, we first remember the following inverse mapping theorem (see [1]):

Theorem 2. Let $E$ and $G$ be two Banach spaces and let $\mathcal{A}: E \mapsto G$ satisfy $\mathcal{A} \in C^{1}(E ; G)$. Assume that $e_{0} \in E$, $\mathcal{A}\left(e_{0}\right)=h_{0}$ and $\mathcal{A}^{\prime}\left(e_{0}\right): E \mapsto G$ is surjective. Then, there exists $\delta>0$ such that, for every $h \in G$ satisfying $\left\|h-h_{0}\right\|_{G}<\delta$, there exists a solution of the equation

$$
\mathcal{A}(e)=h, \quad e \in E \text {. }
$$

In our setting, we use this theorem with the spaces $E=\widetilde{E}_{N}$ and $G=G_{1} \times G_{2}$ with

$$
G_{1}= \begin{cases}L^{2}\left(\beta_{3}(0, T) ; H^{-1}(\Omega)^{2}\right) \times L^{2}\left(\beta_{3}(0, T) ; H^{-1}(\Omega)\right) & \text { if } N=2, \\ L^{2}\left(\beta_{3}(0, T) ; W^{-1,6}(\Omega)^{3}\right) \times L^{2}\left(\beta_{3}(0, T) ; H^{-1}(\Omega)\right) & \text { if } N=3\end{cases}
$$

and

$$
G_{2}= \begin{cases}H \times L^{2}(\Omega) & \text { if } N=2 \\ \left(L^{4}(\Omega)^{3} \cap H\right) \times L^{2}(\Omega) & \text { if } N=3\end{cases}
$$

and the operator

$$
\begin{aligned}
& \mathcal{A}\left(z, q, \rho, v_{1}, v_{2}\right)=\left(\mathcal{A}_{1}\left(z, q, \rho, v_{1}\right), \mathcal{A}_{2}\left(z, \rho, v_{2}\right), z(0), \rho(0)\right), \\
& \mathcal{A}_{1}\left(z, q, \rho, v_{1}\right)=L_{1} z+(z \cdot \nabla) z+\nabla q-v_{1} 1_{\omega}-\rho e_{N}
\end{aligned}
$$

and

$$
\mathcal{A}_{2}\left(z, \rho, v_{2}\right)=L_{2} \rho+z \cdot \nabla \rho+\nabla \cdot(\bar{\theta} z)-v_{2} 1_{\omega}
$$

for every $\left(z, q, \rho, v_{1}, v_{2}\right) \in \widetilde{E}_{N}$.

In the following proposition, we check that the previous framework fits the regularity required to apply Theorem 2.

Proposition 3. Let us assume that $\bar{y} \in L^{\infty}(Q)^{N}$. Then, $\mathcal{A} \in C^{1}(E ; G)$.

Proof. We start by noticing that all the terms arising in the definition of $\mathcal{A}$ are linear (and consequently $C^{1}$ ), except for $(z \cdot \nabla) z$ and $z \cdot \nabla \rho$. However, the operators

$$
\left(\left(z, q, \rho, v_{1}, v_{2}\right),\left(\tilde{z}, \tilde{q}, \tilde{\rho}, \tilde{v}_{1}, \tilde{v}_{2}\right)\right) \longmapsto((z \cdot \nabla) \tilde{z}, z \cdot \nabla \tilde{\rho})
$$

are bilinear, so it suffices to prove their continuity from $\widetilde{E}_{N} \times \widetilde{E}_{N}$ into $G_{1}$.

In fact, for $N=2$ we can use that $\beta_{3}^{1 / 2}(z, \tilde{z}, \tilde{\rho}) \in L^{4}(Q)^{5}$ for any couple $\left(\left(z, q, \rho, v_{1}, v_{2}\right),\left(\tilde{z}, \tilde{q}, \tilde{\rho}, \tilde{v}_{1}, \tilde{v}_{2}\right)\right) \in$ $\widetilde{E}_{N} \times \widetilde{E}_{N}$ and we get

$$
\|(z \cdot \nabla) \tilde{z}\|_{L^{2}\left(\beta_{3}(0, T) ; H^{-1}(\Omega)^{2}\right)} \leqslant C\|z \otimes \tilde{z}\|_{L^{2}\left(\beta_{3}(0, T) ; L^{2}(\Omega)^{2}\right)} \leqslant C\left\|\beta_{3}^{1 / 2} z\right\|_{L^{4}(Q)^{2}}\left\|\beta_{3}^{1 / 2} \tilde{z}\right\|_{L^{4}(Q)^{2}}
$$


and

$$
\|z \cdot \nabla \tilde{\rho}\|_{L^{2}\left(\beta_{3}(0, T) ; H^{-1}(\Omega)\right)} \leqslant C\|\tilde{\rho} z\|_{L^{2}\left(\beta_{3}(0, T) ; L^{2}(\Omega)^{2}\right)} \leqslant C\left\|\beta_{3}^{1 / 2} z\right\|_{L^{4}(Q)^{2}}\left\|\beta_{3}^{1 / 2} \tilde{\rho}\right\|_{L^{4}(Q)} .
$$

On the other hand, for $N=3$ we find that

$$
\begin{aligned}
\|(z \cdot \nabla) \tilde{z}\|_{L^{2}\left(\beta_{3}(0, T) ; W^{-1,6}(\Omega)^{3}\right)} & \leqslant C\|z \otimes \tilde{z} \mid\|_{L^{2}\left(\beta_{3}(0, T) ; L^{6}(\Omega)^{3}\right)} \\
& \leqslant C\left\|\beta_{3}^{1 / 2} z\right\|_{L^{4}\left(0, T ; L^{12}(\Omega)^{3}\right)}\left\|\beta_{3}^{1 / 2} \tilde{z}\right\|_{L^{4}\left(0, T ; L^{12}(\Omega)^{3}\right)}
\end{aligned}
$$

and

$$
\|z \cdot \nabla \tilde{\rho}\|_{L^{2}\left(\beta_{3}(0, T) ; H^{-1}(\Omega)\right)} \leqslant C\|\tilde{\rho} z\|_{L^{2}\left(\beta_{3}(0, T) ; L^{2}(\Omega)^{3}\right)} \leqslant C\left\|\beta_{3}^{1 / 2} z\right\|_{L^{4}\left(0, T ; L^{6}(\Omega)^{3}\right)}\left\|\beta_{3}^{1 / 2} \tilde{z}\right\|_{L^{4}\left(0, T ; L^{3}(\Omega)\right)} .
$$

Therefore, in both cases the continuity of (77) is established.

This proves Proposition 3.

As a consequence of this result, we can apply Theorem 2 for $e_{0}=0 \in \mathbf{R}^{N}$ and $h_{0}=0$. In fact, $\mathcal{A}^{\prime}(0,0,0,0,0)$ : $E \mapsto G$ is given by

$$
\mathcal{A}^{\prime}(0,0,0,0,0)\left(z, q, \rho, v_{1}, v_{2}\right)=\left(L_{1} z+\nabla q-v_{1} 1_{\omega}-\rho e_{N}, L_{2} \rho+\nabla \cdot(\bar{\theta} z)-v_{2} 1_{\omega}, z(0), \rho(0)\right)
$$

for all $\left(z, q, \rho, v_{1}, v_{2}\right) \in \widetilde{E}_{N}$ and is surjective in view of the null controllability result for the linearized system (58) given in Proposition 2.

As a conclusion, an application of Theorem 2 gives the existence of $\delta>0$ such that, if

$$
\|(z(0), \rho(0))\|_{L^{2 N-2}(\Omega)^{N} \times L^{2}(\Omega)} \leqslant \delta,
$$

then we find two controls $\left(v_{1}, v_{2}\right)$ such that the associated solution to (76) verifies $z(T)=0$ and $\rho(T)=0$ in $\Omega$.

This establishes the null controllability result for system (11) and so concludes the proof of Theorem 1.

\section{Appendix}

In this paragraph, we obtain a local estimate of the term on $\Delta \varphi$ appearing in the right-hand side of (31). Let us first introduce two open sets $\omega_{3}$ and $\omega_{4}$ such that $\omega_{2} \Subset \omega_{3} \Subset \omega_{4} \Subset \omega$ and a function $\zeta \in \mathcal{D}\left(\omega_{4}\right)$ with $\zeta \equiv 1$ in $\omega_{3}$.

Secondly, for simplicity we define $\hat{\eta}=s \lambda \mathrm{e}^{-s \hat{\alpha}} \hat{\xi}$ and we set

$$
\tilde{u}(x, t)=\hat{\eta}(t) \zeta(x) \Delta \varphi(x, T-t) \quad \text { in } \mathbf{R}^{N} \times(0, T),
$$

where $u$ has been extended by zero outside $\omega_{4}$.

Let us first see which is the heat equation satisfied by $u$. Indeed, applying Laplace's operator to the equation verified by $\varphi$ and keeping in mind (23), we get

$$
(\Delta \varphi(T-t))_{t}-\Delta(\Delta \varphi(T-t))=\tilde{f} \quad \text { in } Q,
$$

where

$$
\begin{aligned}
\tilde{f}= & \Delta(D \varphi \bar{y})(T-t)+\Delta g_{1}(T-t)+\Delta(\bar{\theta} \nabla \psi)(T-t)-\nabla(\nabla \cdot(D \varphi \bar{y})(T-t)) \\
& -\nabla\left(\nabla \cdot g_{1}(T-t)\right)-\nabla(\nabla \cdot(\bar{\theta} \nabla \psi(T-t))) .
\end{aligned}
$$

From (78), we deduce that

$$
\begin{cases}\tilde{u}_{t}-\Delta \tilde{u}=\widetilde{F} & \text { in } \mathbf{R}^{N} \times(0, T), \\ \tilde{u}(0)=0 & \text { in } \mathbf{R}^{N},\end{cases}
$$


where

$$
\widetilde{F}=\hat{\eta} \zeta \tilde{f}+\hat{\eta}^{\prime} \zeta \Delta \varphi(T-t)-2 \hat{\eta} \nabla \zeta \cdot \nabla \Delta \varphi(T-t)-\hat{\eta} \Delta \zeta \Delta \varphi(T-t) .
$$

Notice that $\widetilde{F} \in L^{2}\left(0, T ; H^{-2}\left(\mathbf{R}^{N}\right)^{N}\right)$ and we a priori know that $\tilde{u} \in L^{2}\left(\mathbf{R}^{N} \times(0, T)\right)^{N}$ (from its definition). From the equation in (79), we have that $\tilde{u}_{t} \in L^{2}\left(0, T ; H^{-2}\left(\mathbf{R}^{N}\right)^{N}\right)$, so that $u(0)$ makes sense.

Now, we rewrite $\widetilde{F}$ in a more appropriate way, so that it is given by the sum of two functions: in the first one, we include all the terms with derivatives of second order of $g_{1}, D \varphi \bar{y}, \bar{\theta} \nabla \psi$ and $\varphi$; in the second one, we consider all the other terms. Notice that this second function has a support contained in $\omega_{4} \backslash \bar{\omega}_{3}$.

More precisely, we put $\widetilde{F}=\widetilde{F}_{1}+\widetilde{F}_{2}$, with

$$
\begin{aligned}
\widetilde{F}_{1}= & \hat{\eta} \Delta(\zeta(D \varphi \bar{y})(T-t))+\hat{\eta} \Delta\left(\zeta g_{1}(T-t)\right)+\hat{\eta} \Delta(\zeta(\bar{\theta} \nabla \psi)(T-t))-\hat{\eta} \nabla(\nabla \cdot(\zeta(D \varphi \bar{y})(T-t))) \\
& -\hat{\eta} \nabla\left(\nabla \cdot\left(\zeta g_{1}(T-t)\right)\right)-\hat{\eta} \nabla(\nabla \cdot(\zeta(\bar{\theta} \nabla \psi)(T-t)))+\hat{\eta}^{\prime} \Delta(\zeta \varphi(T-t))
\end{aligned}
$$

and

$$
\begin{aligned}
F_{2}= & -2 \hat{\eta} \nabla \zeta \cdot \nabla(D \varphi \bar{y})(T-t)-\hat{\eta} \Delta \zeta(D \varphi \bar{y})(T-t)-2 \hat{\eta} \nabla \zeta \cdot \nabla g_{1}(T-t)-\hat{\eta} \Delta \zeta g_{1}(T-t) \\
& -2 \hat{\eta} \nabla \zeta \cdot \nabla(\bar{\theta} \nabla \psi)(T-t)-\hat{\eta} \Delta \zeta(\bar{\theta} \nabla \psi)(T-t)+\hat{\eta} \nabla(\nabla \zeta \cdot(D \varphi \bar{y})(T-t)) \\
& +\hat{\eta} \nabla \zeta(\nabla \cdot(D \varphi \bar{y})(T-t))+\hat{\eta} \nabla\left(\nabla \zeta \cdot g_{1}(T-t)\right)+\hat{\eta} \nabla \zeta\left(\nabla \cdot g_{1}(T-t)\right)+\hat{\eta} \nabla(\nabla \zeta \cdot(\bar{\theta} \nabla \psi)(T-t)) \\
& +\hat{\eta} \nabla \zeta(\nabla \cdot(\bar{\theta} \nabla \psi)(T-t))-2 \hat{\eta}^{\prime} \nabla \zeta \cdot \nabla \varphi(T-t)-\hat{\eta}^{\prime} \Delta \zeta \varphi(T-t) \\
& -2 \hat{\eta} \nabla \zeta \cdot \nabla \Delta \varphi(T-t)-\hat{\eta} \Delta \zeta \Delta \varphi(T-t) .
\end{aligned}
$$

Notice that $\widetilde{F}, \widetilde{F}_{1} \in L^{2}\left(0, T ; H^{-2}\left(\mathbf{R}^{N}\right)^{N}\right)$, while $\widetilde{F}_{2} \in L^{2}\left(0, T ; H^{-1}\left(\mathbf{R}^{N}\right)^{N}\right)$. Next, we are going to introduce two functions $\tilde{u}^{1}$ and $\tilde{u}^{2}$ in $L^{2}\left(\mathbf{R}^{N} \times(0, T)\right)^{N}$ satisfying

$$
\begin{cases}\tilde{u}_{t}^{i}-\Delta \tilde{u}^{i}=\widetilde{F}_{i} & \text { in } \mathbf{R}^{N} \times(0, T), \\ \tilde{u}^{i}(0)=0 & \text { in } \mathbf{R}^{N}\end{cases}
$$

for $i=1,2$. Once this is made, we will have $\tilde{u}=\tilde{u}^{1}+\tilde{u}^{2}$ and it will suffice to estimate the integrals

$$
\iint_{\omega_{2} \times(0, T)}\left|\tilde{u}^{i}\right|^{2} \mathrm{~d} x \mathrm{~d} t .
$$

Definition and estimate of $\tilde{u}^{1}$. By definition, we will say that $\tilde{u}^{1}$ is the solution by transposition of the Cauchy problem for the heat equation (80) for $i=1$. This means that $\tilde{u}^{1}$ is the unique function in $L^{2}\left(\mathbf{R}^{N} \times(0, T)\right)^{N}$ that, for each $h \in L^{2}\left(\mathbf{R}^{N} \times(0, T)\right)^{N}$, one has

$$
\begin{aligned}
& \iint_{\mathbf{R} \times(0, T)} \tilde{u}^{1} \cdot h \mathrm{~d} x \mathrm{~d} t=\iint_{\mathbf{R}^{N} \times(0, T)}\left(\hat{\eta} \zeta\left(g_{1}+D \varphi \bar{y}+\bar{\theta} \nabla \psi\right)(T-t)\right) \cdot \Delta z \mathrm{~d} x \mathrm{~d} t
\end{aligned}
$$

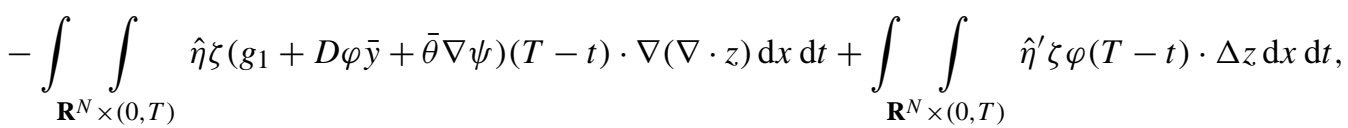

where $z$ is the solution of

$$
\begin{cases}-z_{t}-\Delta z=h & \text { in } \mathbf{R}^{N} \times(0, T) \\ z(T)=0 & \text { in } \mathbf{R}^{N}\end{cases}
$$

Remark that, for every $h \in L^{2}\left(\mathbf{R}^{N} \times(0, T)\right)^{N}$, (82) possesses exactly one solution $z \in L^{2}\left(0, T ; H^{2}\left(\mathbf{R}^{N}\right)^{N}\right)$ that depends continuously on $h$. Therefore, $\tilde{u}_{1}$ is well defined and

$$
\left\|\tilde{u}^{1}\right\|_{L^{2}\left(\mathbf{R}^{N} \times(0, T)\right)^{N}} \leqslant \widehat{C}_{1}\left\|\widetilde{F}_{1}\right\|_{L^{2}\left(0, T ; H^{-2}\left(\mathbf{R}^{N}\right)^{N}\right)},
$$


for a positive constant $\widehat{C}_{1}$. Furthermore, it is not difficult to show that $\tilde{u}^{1} \in C^{0}\left([0, T] ; H^{-2}\left(\mathbf{R}^{N}\right)^{N}\right)$ and solves (80) for $i=1$ in the distributional sense.

Furthermore, we can easily deduce from (83) that

$$
\begin{aligned}
& \iint_{\mathbf{R}^{N} \times(0, T)}\left|\tilde{u}^{1}\right|^{2} \mathrm{~d} x \mathrm{~d} t \leqslant C\left(\iint_{\mathbf{R}^{N} \times(0, T)}\left|\hat{\eta} \zeta g_{1}\right|^{2} \mathrm{~d} x \mathrm{~d} t\right. \\
& \left.\quad+\int_{\mathbf{R}^{N} \times(0, T)}|\hat{\eta} \zeta \bar{\theta} \nabla \psi|^{2} \mathrm{~d} x \mathrm{~d} t+\iint_{\mathbf{R}^{N} \times(0, T)}|\hat{\eta} \zeta D \varphi \bar{y}|^{2} \mathrm{~d} x \mathrm{~d} t+\iint_{\mathbf{R}^{N} \times(0, T)}\left|\hat{\eta}^{\prime} \zeta \varphi\right|^{2} \mathrm{~d} x \mathrm{~d} t\right),
\end{aligned}
$$

for a constant $C>0$. Here, we have used the fact that

$$
\hat{\eta}(T-t)=\hat{\eta}(t) \quad \forall t \in(0, T) .
$$

Thanks to the properties of $\zeta$, we finally get

$$
\begin{aligned}
\iint_{\omega_{2} \times(0, T)}\left|\tilde{u}^{1}\right|^{2} \mathrm{~d} x \mathrm{~d} t \leqslant & \iint_{\mathbf{R}^{N} \times(0, T)}\left|\tilde{u}^{1}\right|^{2} \mathrm{~d} x \mathrm{~d} t \leqslant C\left(\iint_{\omega_{4} \times(0, T)}\left|\hat{\eta} g_{1}\right|^{2} \mathrm{~d} x \mathrm{~d} t+\iint_{\omega_{4} \times(0, T)}\left|\hat{\eta}^{\prime} \varphi\right|^{2} \mathrm{~d} x \mathrm{~d} t\right. \\
& \left.+\iint_{\omega_{4} \times(0, T)}|\hat{\eta} D \varphi \bar{y}|^{2} \mathrm{~d} x \mathrm{~d} t+\iint_{\omega_{4} \times(0, T)}|\hat{\eta} \bar{\theta} \nabla \psi|^{2} \mathrm{~d} x \mathrm{~d} t\right),
\end{aligned}
$$

with $C>0$.

Definition and estimate of $\tilde{u}^{2}$. Now, we deal with the Cauchy problem (80) for $i=2$, where the right-hand side is in $L^{2}\left(0, T ; H^{-1}\left(\mathbf{R}^{N}\right)^{N}\right)$. The existence and uniqueness of a solution $u^{2} \in L^{2}\left(0, T ; H^{1}\left(\mathbf{R}^{N}\right)^{N}\right)$ is classical. Recall that $\widetilde{F}_{1}(t)$ has support in $\omega_{4} \backslash \bar{\omega}_{3}$ for $t$ a.e., while we would like to estimate the $L^{2}$-norm of the solution in $\omega_{2}$ and $\omega_{2}$ is disjoint of $\omega_{4} \backslash \bar{\omega}_{3}$.

We will start by writing $\tilde{u}^{2}$ in terms of the fundamental solution $G=G(x, t)$ of the heat equation. To do this, we first notice that $\widetilde{F}_{2}$ can be written in the form

$$
\widetilde{F}_{2}=\widetilde{F}_{21}+\nabla \cdot \widetilde{F}_{22},
$$

where $\widetilde{F}_{21}$ and $\widetilde{F}_{22}$ are $L^{2}$ functions supported by $\left(\omega_{4} \backslash \bar{\omega}_{3}\right) \times[0, T]$ which can be written as sums of derivatives up to the second order of products $\hat{\eta} D^{\beta} \zeta g_{1}, \hat{\eta} D^{\beta} \zeta \varphi, \hat{\eta} D^{\beta} \zeta D \varphi \bar{y}, \hat{\eta} D^{\beta} \zeta \bar{\theta} \nabla \psi$ and $\hat{\eta}^{\prime} D^{\beta} \zeta \varphi$ with $1 \leqslant|\beta| \leqslant 4$.

Observe that, for any $y \in \omega_{4} \backslash \bar{\omega}_{3}$ and any $x \in \omega_{2}$, one has $|x-y| \geqslant \operatorname{dist}\left(\partial \omega_{3}, \partial \omega_{4}\right)=d>0$. Then, we have:

$$
\tilde{u}^{2}(x, t)=\int_{0}^{t} \int_{\omega_{4} \backslash \bar{\omega}_{3}} G(x-y, t-s) \widetilde{F}_{21}(y, s) \mathrm{d} y \mathrm{~d} s-\int_{0}^{t} \int_{\omega_{4} \backslash \bar{\omega}_{3}} \nabla_{y} G(x-y, t-s) \cdot \widetilde{F}_{22}(y, s) \mathrm{d} y \mathrm{~d} s
$$

for all $(x, t) \in \omega_{2} \times(0, T)$, where

$$
G(x, t)=\frac{1}{(4 \pi t)^{N / 2}} \mathrm{e}^{-|x|^{2} / 2 t} \quad \forall x \in \mathbf{R}^{N}, \forall t>0 .
$$

Now we integrate by parts with respect to $y$ in (85), passing all the derivatives from $\widetilde{F}_{21}$ and $\widetilde{F}_{22}$ to $G$ and $\nabla_{y} G$. This is possible because we are integrating in a region where $G$ is of class $C^{\infty}$. This yields an expression for $u^{2}$ of the form

$$
\tilde{u}^{2}(x, t)=\iint_{\left(\omega_{4} \backslash \overline{\omega_{3}}\right) \times(0, t)} \sum_{\alpha \in I, \beta \in J} D_{y}^{\alpha} G(x-y, t-s) D_{y}^{\beta} \zeta(y) z_{\alpha, \beta}(y, s) \mathrm{d} y \mathrm{~d} s,
$$


where all $\alpha \in I$ satisfy $|\alpha| \leqslant 3$, all $\beta \in J$ satisfy $1 \leqslant|\beta| \leqslant 4$ and

$$
\begin{aligned}
z_{\alpha, \beta}(y, s)= & \hat{\eta}(s)\left(C_{\alpha, \beta} g_{1}(y, s)+D_{\alpha, \beta} \varphi(y, s)+E_{\alpha, \beta}(D \varphi \bar{y})(y, s)\right. \\
& \left.+F_{\alpha, \beta}(\bar{\theta} \nabla \psi)(y, s)\right)+G_{\alpha, \beta} \hat{\eta}^{\prime}(s) \varphi(y, s), \quad C_{\alpha, \beta}, D_{\alpha, \beta}, E_{\alpha, \beta}, F_{\alpha, \beta}, G_{\alpha, \beta} \in \mathbf{R} .
\end{aligned}
$$

From the previous considerations, we readily have

$$
\left|\tilde{u}^{2}(x, t)\right| \leqslant \iint_{\left(\omega_{4} \backslash \bar{\omega}_{3}\right) \times(0, t)} \sum_{\alpha \in I}\left|D_{y}^{\alpha} G(x-y, t-s)\right||z(y, s)| \mathrm{d} y \mathrm{~d} s
$$

for all $(x, t) \in \omega_{2} \times(0, T)$, where

$$
z(y, s)=\hat{\eta}(s)\left(C_{1} g_{1}(y, s)+C_{2} \varphi(y, s)+C_{3}(D \varphi \bar{y})(y, s)+C_{4}(\bar{\theta} \nabla \psi)(y, s)\right)+C_{5} \hat{\eta}^{\prime}(s) \varphi(y, s) .
$$

Obviously, for every $0<\delta<d$ there exists a positive constant $C(\delta, \omega)$ such that

$$
\left|D^{\alpha} G(x-y, t-s)\right| \leqslant C \exp \left(\frac{-\delta^{2}}{2(t-s)}\right)
$$

for all $(x, t) \in \omega_{2} \times(0, T)$, all $(y, s) \in\left(\omega_{4} \backslash \overline{\omega_{3}}\right) \times(0, t)$ and any $\alpha \in I$, so that

$$
\left|\tilde{u}^{2}(x, t)\right| \leqslant \widehat{C} \iint_{\left(\omega_{2} \backslash \bar{\omega}_{3}\right) \times(0, t)} \exp \left(\frac{-\delta^{2}}{2(t-s)}\right)|z(y, s)| \mathrm{d} y \mathrm{~d} s
$$

with $C(\omega)>0$.

At this moment, we integrate in $\omega_{2} \times(0, T)$ and we obtain:

$$
\begin{aligned}
\iint_{\omega_{2} \times(0, T)}\left|\tilde{u}^{2}\right|^{2} \mathrm{~d} x \mathrm{~d} t & \leqslant C \int_{0}^{T}\left(\int_{0}^{t} \int_{\omega_{4} \backslash \bar{\omega}_{3}} \exp \left(\frac{-\delta^{2}}{2(t-s)}\right)|z(y, s)| \mathrm{d} y \mathrm{~d} s\right)^{2} \mathrm{~d} t \\
& \leqslant C T \int_{0}^{T}\left(\int_{0}^{t} \exp \left(\frac{-\delta^{2}}{2(t-s)}\right)\|z(s)\|_{L^{2}\left(\omega_{4}\right)}^{2} \mathrm{~d} s\right) \mathrm{d} t
\end{aligned}
$$

for some $\widehat{C}(\omega)>0$.

Finally, we write the last term as a convolution, say

$$
\int_{0}^{T}\left(\tilde{f}_{1} * \tilde{f}_{2}\right)(t) \mathrm{d} t
$$

where

$$
\tilde{f}_{1}(t)=\mathrm{e}^{-\delta^{2} / t} 1_{(0,+\infty)}(t) \in L^{1}(\mathbf{R}), \quad \tilde{f}_{2}(t)=\|z(t)\|_{L^{2}\left(\omega_{4}\right)}^{2} 1_{[0, T]}(t) \in L^{1}(\mathbf{R})
$$

and we use Young's inequality. This provides

$$
\iint_{\omega_{2} \times(0, T)}\left|\tilde{u}^{2}\right|^{2} \mathrm{~d} x \mathrm{~d} t \leqslant C T \int_{\omega_{4} \times(0, T)}|z|^{2} \mathrm{~d} x \mathrm{~d} t .
$$

Taking into account the expression of $z$, we get 


$$
\begin{aligned}
\iint_{\omega_{2} \times(0, T)}\left|\tilde{u}^{2}\right|^{2} \mathrm{~d} x \mathrm{~d} t \leqslant & C T\left(\int_{\omega_{4} \times(0, T)}\left|\hat{\eta}^{\prime} \varphi\right|^{2} \mathrm{~d} x \mathrm{~d} t\right. \\
& \left.+\iint_{\omega_{4} \times(0, T)}|\hat{\eta}|^{2}\left(\left|g_{1}\right|^{2}+|D \varphi \bar{y}|^{2}+|\bar{\theta} \nabla \psi|+|\varphi|^{2}\right) \mathrm{d} x \mathrm{~d} t\right) .
\end{aligned}
$$

Putting this together with (84), we arrive at the estimate searched in this paragraph:

$$
\begin{aligned}
\iint_{\omega_{2} \times(0, T)}|\hat{\eta}|^{2}|\Delta \varphi|^{2} \mathrm{~d} x \mathrm{~d} t \leqslant & C(1+T)\left(\int_{\omega_{4} \times(0, T)}\left|\hat{\eta}^{\prime}\right|^{2}|\varphi|^{2} \mathrm{~d} x \mathrm{~d} t\right. \\
& \left.+\iint_{\omega_{4} \times(0, T)}|\hat{\eta}|^{2}\left(\left|g_{1}\right|^{2}+|D \varphi \bar{y}|^{2}+|\bar{\theta} \nabla \psi|+|\varphi|^{2}\right) \mathrm{d} x \mathrm{~d} t\right) .
\end{aligned}
$$

\section{References}

[1] V.M. Alekseev, V.M. Tikhomirov, S.V. Fomin, Optimal Control, Contemp. Soviet Math., Consultants Bureau, New York, 1987. Translated from the Russian by V.M. Volosov.

[2] E. Fernández-Cara, S. Guerrero, Global Carleman inequalities for parabolic systems and applications to controllability, SIAM J. Control Optimization, in press.

[3] E. Fernández-Cara, S. Guerrero, O.Yu. Imanuvilov, J.-P. Puel, Local exact controllability of the Navier-Stokes system, J. Math. Pures Appl. 83 (12) (2004) 1501-1542.

[4] A.V. Fursikov, O.Yu. Imanuvilov, Controllability of Evolution Equations, Lecture Notes, vol. 34, Seoul National University, Korea, 1996.

[5] A.V. Fursikov, O.Yu. Imanuvilov, Local exact boundary controllability of the Boussinesq equation, SIAM J. Control Optim. 36 (2) (1998) 391-421.

[6] A.V. Fursikov, O.Yu. Imanuvilov, Exact controllability of the Navier-Stokes and Boussinesq equations, Uspekhi Mat. Nauk 54 (3) (327) (1999) 93-146 (in Russian). Translation in Russian Math. Surveys 54 (3) (1999) 565-618.

[7] Y. Giga, H. Sohr, Abstract $L^{p}$ estimates for the Cauchy problem with applications to the Navier-Stokes equations in exterior domains, J. Funct. Anal. 102 (1991) 72-94.

[8] O.Yu. Imanuvilov, Remarks on exact controllability for the Navier-Stokes equations, ESAIM Control Optim. Calc. Var. 6 (2001) $39-72$.

[9] O.Yu. Imanuvilov, J.-P. Puel, Global Carleman estimates for weak elliptic non homogeneous Dirichlet problem, Int. Math. Res. Notices 16 (2003) 883-913.

[10] O.A. Ladyzenskaya, A.V. Solonnikov, N.N. Uraltzeva, Linear and Quasilinear Equations of Parabolic Type, Transl. Math. Monographs, vol. 23, 1967.

[11] R. Temam, Navier-Stokes Equations. Theory and Numerical Analysis, Stud. Math. Appl., vol. 2, North-Holland, Amsterdam, 1977. 